\title{
Scattering Theory for the Elastic Wave Equation
}

\author{
By \\ Yoshihiro ShibAtA* and Hideo SogA**
}

\section{§ 0 . Introduction}

Let us set

$$
L u(x)=\sum_{i, j=1}^{n} \partial_{i}\left(a_{i j}(x) \partial_{j} u(x)\right), \partial_{i}=\partial / \partial x_{i}, u={ }^{t}\left(u_{1}, \cdots, u_{n}\right),
$$

where $a_{i j}(x)$ are $n \times n$ martices of real-valued functions $\in \mathcal{B}^{\infty}\left(\boldsymbol{R}^{n}\right)(=\{f \in$ $C^{\infty}\left(\boldsymbol{R}^{n}\right)\left|\sup _{x \in \boldsymbol{R}^{n}}\right| \partial_{x}^{\alpha} f(x) \mid<\infty$ for any multi-index $\left.\left.\alpha\right\}\right)$. Let $\Omega$ be an exterior domain in $\boldsymbol{R}^{n}$ whose boundary $\partial \Omega$ is a compact and $C^{\infty}$ hypersurface contained in $\left\{x \in \boldsymbol{R}^{n}|| x \mid<r_{0}\right\}$. We consider the elastic wave equation:

$$
\left\{\begin{array}{l}
\left(\partial_{t}^{2}-L\right) u(t, x)=0 \text { in } \boldsymbol{R} \times \Omega\left(\partial_{t}=\partial / \partial t\right), \quad B u\left(t, x^{\prime}\right)=0 \text { on } \boldsymbol{R} \times \partial \Omega \\
u(0, x)=f_{1}(x) \text { in } \Omega, \quad \partial_{t} u(0, x)=f_{2}(x) \text { in } \Omega .
\end{array}\right.
$$

Here the boundary operator $B$ is of the form

$$
\begin{aligned}
& B u=\left.u\right|_{\partial \Omega}, \quad \text { or } \\
& B u=\left.\sum_{i, j=1}^{n} \nu_{i}(x) a_{j i}(x) \partial_{j} u\right|_{\partial \Omega},
\end{aligned}
$$

where $\nu(x)=\left(\nu_{1}(x), \cdots, \nu_{n}(x)\right)$ denotes the unit outer normal to $\partial \Omega$ at $x \in \partial \Omega$. Lax and Phillips $[6,7]$ formulated the scattering theory for the scalar-valued wave equation. The purpose of this paper is to make an analogous formulation for the elastic wave equation (0.1).

Throughout this paper, we assume that the space dimension $n \geqq 3$. Furthermore, it is assumed that$$
a_{i p j q}(x)=a_{p i j q}(x)=a_{j q i p}(x),
$$$$
i, j, p, q=1, \cdots, n \text { (hyperelasticity), }
$$

Comnunicated by S. Matsuura, April 16, 1988.

* Institute of Mathematics, University of Tsukuba, Ibaraki 305, Japan.

** Faculty of Education, Ibaraki University, Ibaraki 310, Japati. 


$$
\sum_{i, j, p, q=1}^{n} a_{i p j q}(x) \varepsilon_{j q} \overline{\varepsilon_{i p}} \geqq \delta_{1} \sum_{i, p=1}^{n}\left|\varepsilon_{i p}\right|^{2} \quad \text { (stability), }
$$

where $a_{i p j q}$ denote the $(p, q)$ components of the $n \times n$ matrix $a_{i j},\left(\varepsilon_{z p}\right)$ is any $n \times n$ Hermitian matrix and $\delta_{1}$ is some positive constant independent of $\varepsilon_{i p}$.

Lax and Phillips $[6,7]$ employed the following energy for the data $f=\left(f_{1}, f_{2}\right)$ :

$$
\frac{1}{2} \int_{\Omega}\left(\left|\nabla_{x} f_{1}\right|^{2}+\left|f_{2}\right|^{2}\right) d x \quad\left(\nabla_{x} f_{1}=\left(\partial_{1} f_{1}, \cdots, \partial_{n} f_{1}\right)\right) .
$$

This played an essential role on their formulation. For the elastic equation, the energy is defined (in physics) by

$$
\frac{1}{2} \int_{\Omega}\left\{\sum_{i, j, p, q=1}^{n} a_{i p j q} \varepsilon_{j q}\left(f_{1}\right) \overline{\varepsilon_{i p}\left(f_{1}\right)}+\left|f_{2}\right|^{2}\right\} d x,
$$

where $f_{s}={ }^{t}\left(f_{i 1}, \cdots, f_{i n}\right), i=1,2$, and $\varepsilon_{i p}\left(f_{1}\right)=\frac{1}{2}\left(\partial_{i} f_{1 p}+\partial_{p} f_{1 i}\right)$ called the stress tensor. By (A.1) we see that this energy is equal to

$$
\|f\|_{E, \Omega}^{2}=\frac{1}{2} \int_{\Omega}\left(\sum_{i, p, j, q=1}^{n} a_{i p j q} \partial_{j} f_{1 q} \overline{\partial_{i} f_{1 p}}+\left|f_{2}\right|^{2}\right) d x .
$$

From (A.2) it follows that

$$
\|f\|_{E, \Omega}^{2} \geqq \delta_{1} \sum_{i, p=1}^{n}\left\|\varepsilon_{i p}\left(f_{1}\right)\right\|_{L^{2}(\Omega)}^{2}+\left\|f_{2}\right\|_{L^{2}(\Omega)}^{2},
$$

and then the energy is non-negative for any $f=\left(f_{1}, f_{2}\right)$. However, it does not mean that $\left\|f^{\prime}\right\|_{E, \Omega}$ is a norm equivalent to $\left\|\partial_{x}^{1} f_{1}\right\|_{L^{2}(\Omega)}+\left\|f_{Z^{2}}\right\|_{L^{2}(\Omega)}\left(\partial_{x}^{1} f_{1}={ }^{t}\left(\nabla_{x} f_{11}, \cdots\right.\right.$, $\left.\nabla_{x} f_{1 n}\right)$ ). To prove this equivalence, we need to verify the inequality:

$$
\sum_{i, p=1}^{n}\left\|\varepsilon_{i p}\left(f_{1}\right)\right\|_{L^{2}(\Omega)}^{2} \geqq \delta_{2}\left\|\partial_{x}^{1} f_{1}\right\|_{L^{2}(\Omega)}^{2},
$$

where $\delta_{2}$ is some positive constant independent of $f_{1}$. The estimate $(0.4)$ is the key to the construction of the scattering theory for the elastic equation. When the domain $\Omega$ is bounded and the boundary condition is the displacement one (the case (0.2)), (0.4) is well-known as Korn's first inequality (cf. $\S 3$ of Duvaut and Lions [1]). We shall give the proof of (0.4) in $\S 1$ below. K.O. Friedrichs [2] also proved Korn's first inequality in the exterior domain. But the function spaces are quite different, and our result cannot follow from his.

We introduce the Hilbert space $H$ of the data $f=\left(f_{1}, f_{2}\right)$ defined as the completion of $\left\{f \in C^{\infty}(\bar{\Omega}) \mid B f_{1}=0\right.$ on $\partial \Omega$, supp $[f]$ is bounded $\}$ in the norm $\|f\|_{E, \Omega}$. This space $H$ coincides with $H_{B}^{1}(\Omega) \times L^{2}(\Omega)$, where $H_{B}^{1}(\Omega)$ is defined by 


$$
\begin{array}{r}
H_{B}^{1}(\Omega)=\left\{\left.u \in H_{\mathrm{loc}}^{1}(\Omega)\left|\partial_{x}^{1} u \in L^{2}(\Omega), \lim _{R \rightarrow \infty} R^{-2} \int_{R \leqq|x| \leqq 2 R}\right| u(x)\right|^{2} d x=0,\right. \\
\left.\left.u\right|_{\partial \Omega}=0 \text { on } \partial \Omega \text { only when } B u=\left.u\right|_{\partial \Omega}\right\} .
\end{array}
$$

$H_{B}^{1}(\Omega)$ is complete in the norm $\left\|\partial_{x}^{1} u\right\|_{L^{2}(\Omega)}$ (c). Corollary 1.6 and Theorem 1.9 in $\S 1$ below). We define

$$
H_{B}^{2}(\Omega)=\left\{u \in H_{B}^{1}(\Omega) \mid \partial_{x}^{1} u \in H^{1}(\Omega), B u=0 \text { on } \partial \Omega\right\} .
$$

Set

$$
A=\left[\begin{array}{ll}
0 & I \\
L & 0
\end{array}\right], \quad v(t)=\left[\begin{array}{l}
u(t, \cdot) \\
\partial_{t} u(t, \cdot)
\end{array}\right] .
$$

Then the equation (0.1) is transformed into

$$
\frac{d v(t)}{d t}=A v(t), \quad v(0)=f
$$

The domain of $A$ is defined by $D(A)=H_{B}^{2}(\Omega) \times\left(H_{B}^{1}(\Omega) \cap L^{2}(\Omega)\right)$. Then, $A$ is a closed operator in $H$; furthermore, $A$ is skew self-adjoint in $H$ (cf. Theorem 1.11 in $\S 1$ below). Therefore, by Stone's theorem (cf. Appendix I in Lax and Phillips [6]), we see that $A$ generates a group $\{U(t)\}_{t \in R}$ of unitary operators on $H$, and that for any $f \in D(A) v(t)=U(t) f$ becomes a $H$-valued $C^{1}$ function and the solution of (0.6).

Using the Radon transformation: $u(x) \rightarrow \tilde{u}(s, \omega)$, Lax and Phillips [6,7] constructed concretely the translation representations for the scalar-valued wave equation. In $\S 2$, we shall construct the analogous representations for (0.1) in the unperturbed case (i.e., $\Omega=\mathbb{R}^{n}$, and $a_{i j}=a_{i j}^{0}$ are constant) and study their properties under an additional assumption:

$$
\begin{aligned}
& L^{0}(\xi)=\sum_{i, j=1}^{n} a_{i j}^{0} \xi_{i} \xi_{j} \text { has eigenvalues of constant } \\
& \text { multiplicity for } \xi \in \mathbb{R}^{n}-\{0\}
\end{aligned}
$$

In the elastic wave equation case, there exist waves of the different modes (i.e., $L^{0}(\xi)$ may have different eigenvalues). We need to notice this phenomenon when defining the translation representations and studying their properties; however, in this process we do not encounter a serious difficulty caused by that phenomenon. The idea of our methods is essentially the same as in Chapter VI of Lax and Phillips [6], which deals with the scattering for symmetric hyperbolic systems of first order. 
In $\S 3$, assuming that the coefficients of $L$ are constant, we shall consider the scattering for $(0.1)$. Set

$$
D_{ \pm}=\left\{f \in H \mid \operatorname{supp}[U(t) f(x)] \subset\left\{(t, x)\left| \pm \eta t+r_{0} \leqq\right| x \mid\right\} \text { if } \pm t>0\right\},
$$

where $\eta^{2}(\eta>0)$ is the minimum of the eigenvalues of $L^{0}(\omega)\left(\omega \in S^{n-1}\right)$. Then it is seen from the discussion in the unperturbed case (in $\S 2$ ) that $D_{+}$(resp. $D_{-}$) has the properties of the outgoing (resp. incoming) subspace for $U(t)$, except

$$
\overline{\bigcup_{t} U(t) D_{ \pm}}=H \text {. }
$$

By this equality we can construct the translation representations for $U(t)$, and also can derive the completeness of the wave operators in the same way as in Lax and Phillips [6,7]. Thus, the main task in $\S 3$ is to prove $(0.7)$. The proof of $(0.7)$ can be reduced to verifying non-existence of the point spectrums of $A$ (cf. $\S 2$ of Chapter $\mathrm{V}$ in Lax and Phillips [6]), and therefore we have only to show that there exist no eigen-functions of $A$ in the domain $D(A)$ (cf. Theorem 3.5 in $\$ 3$ below). The key to this show is to prove that if $f \in D(A)$ satisfies $(A-i \sigma) f(x)=0$ for every large $|x|$, then $f(x)=0$ for every large $|x|$. The methods in Lax and Phillips [6] are not applicable to this proof in our case. Multiplying $A-i \sigma I$ by its cofactor, we transform it into a diagonal operator and carry out the proof by means of a uniqueness theorem for single equations of higher order obtained in Littman [8], Hörmander [4] and Murata [10].

Yamamoto [14] makes a related study. He considers the isotropic equation in three dimensional space (i.e., $a_{i p j q}(x)=\mu\left(\delta_{p q} \delta_{i j}+\delta_{i q} \delta_{j p}\right)+\lambda \delta_{i p} \delta_{j q}, \delta_{i p}$ being Kronecker's delta; $\lambda$ and $\mu$ being the Lamé constants, and $n=3$ ) with the displacement boundary condition (the case $(0.2)$ ). which is contained in our case. And then, he obtains the same results as ours. But it seems difficult to apply his methods to the traction boundary condition case (the case (0.3)). For, he does not derive the estimate (0.4), which is essential in the traction boundary condition case.

We note that Iwashita and Shibata [5] investigate the analyticity of spectral functions of $L+\sigma^{2} I$ and the rate of the local energy decay of the solutions to $(0.1)$.

\section{$\S 1$. Spaces of the Data and Properties of the Generator $\mathbb{A}$}

In what follows, the Roman letters $u, v, w$ and the Greek letters $\phi, \psi$ are used to denote $n$-dimensional row vector and scalar-valued functions, respectively. 
For any domain $G$ in $\boldsymbol{R}^{n}, C_{0}^{\infty}(G)$ denotes the space of all $C^{\infty}$ functions on $\boldsymbol{R}^{n}$ whose supports are compact and lie in $G . C_{0}^{\infty}(\bar{G})$ denotes the space of all functions in $C^{\infty}(\bar{G})$ whose supports are compact. In particular, functions in $C_{0}^{\infty}(G)$ vanish near the boundary of $G$. By $H^{m}(G)$ we denote the Sobolev space of order $m$ on $G$; put $H_{\mathrm{loc}}^{m}(G)=\left\{\phi \mid \psi \phi \in H^{m}(G)\right.$ for any $\left.\psi \in C_{0}^{\infty}\left(\boldsymbol{R}^{n}\right)\right\}$. Set $\Omega_{R}=\{x \in \Omega|| x \mid<R\}\left(R \geqq r_{0}\right)$. For any space $\Gamma$ of scalar valued functions we abbreviate the product space $\Gamma \times \cdots \times \Gamma$ by also $\Gamma$. $\quad \chi(x)$ will always refer to a real and scalar valued function in $C_{0}^{\infty}\left(\boldsymbol{R}^{n}\right)$ such that $0 \leqq \chi \leqq 1, \chi(x)=1$ for $|x| \leqq 1$ and $=0$ for $|x| \geqq 2$.

As is stated in $\S 0$, the purpose of this section is to discuss the skew selfadjointness of the operator $A$ given in (0.5). Set

$\dot{H}^{m}(\Omega)=\left\{u \in H_{\mathrm{loc}}^{m}(\Omega) \mid \partial_{x}^{\alpha} u \in L^{2}(\Omega)\right.$ for any multi-index $\alpha$ with $1 \leqq|\alpha| \leqq m$,

$$
\left.\lim _{R \rightarrow \infty} R^{-2} \int_{R \leqq|x| \leqq 2 R}|u(x)|^{2} d x=0\right\} .
$$

Put

$$
(u, v)_{m, \Omega}=\sum_{1 \leqq|\alpha| \leqq m} \sum_{j=1}^{n} \int_{\Omega} \partial_{x}^{\alpha} u_{j}(x) \overline{\partial_{x}^{\alpha} v_{j}(x)} d x,|u|_{m, \Omega}^{2}=(u, u)_{m, \Omega}
$$

for any $u={ }^{t}\left(u_{1}, \cdots, u_{n}\right)$ and $v={ }^{t}\left(v_{1}, \cdots, v_{n}\right) \in \dot{H}^{m}(\Omega)$ ( $m$ being integers $\left.\geqq 1\right)$.

Theorem 1.1. Let $m$ be an integer $\geqq 1$. Then, $\dot{H}^{m}(\Omega)$ is a Hilbert space equipped with the innerproduct $(,)_{m, \Omega}$. Furthermore, $C_{0}^{\infty}(\bar{\Omega})$ is dense in $\dot{H}^{m}(\Omega)$.

To prove Theorem 1.1, we need the following three technical lemmas.

Lemma 1.2. For any $u \in \dot{H}^{m}(\Omega)(m \geqq 1)$ and $R \geqq r_{0}$, there exists a sequence $\left\{u_{k, R}\right\}_{k=1,2, \cdots} \subset C_{0}^{\infty}(\bar{\Omega})$ such that

$$
\left|u_{k, R}-u\right|_{m, \Omega} \rightarrow 0 \text { and }\left\|u_{k, R}-u\right\|_{L^{2}\left(\Omega_{R}\right)} \rightarrow 0 \text { as } k \rightarrow \infty \text {. }
$$

Lemma 1.3. For any $u \in \dot{H}^{1}(\Omega)$ and $R \geqq r_{0}+1$,

$$
\int_{|x| \geqq R}|u(x)|^{2}|x|^{-2} d x \leqq 4 \int_{|x| \geqq R}\left|\partial_{x}^{1} u(x)\right|^{2} d x .
$$

Lemma 1.4. There exists a constant $C>0$ independent of $R \geqq r_{0}$ such that

$$
\|u\|_{L^{2}\left(\Omega_{R}\right)} \leqq C R\left\|\partial_{x}^{1} u\right\|_{L^{2}(\Omega)} \quad \text { for any } u \in \dot{H}^{1}(\Omega) \text { and } R \geqq r_{0} .
$$

Deferring the proofs of Lemmas 1.2-1.4, we give

Proof of Theorem 1.1. From Lemma 1.4 we see that if $(u, u)_{m, \Omega}=0$, then 
$u=0$. Thus, we can easily check that $(u, v)_{m, \Omega}$ is an innerproduct. In view of Lemma 1.2 , for any $u \in \dot{H}^{m}(\Omega)$ we can choose a sequence $\left\{u_{k, r_{0}}\right\}_{k=1,2, \ldots} \subset C_{0}^{\infty}(\bar{\Omega})$ so that $\left|u_{k, r_{0}}-u\right|_{m, \Omega} \rightarrow 0$ as $k \rightarrow \infty$, from which it follows that $C_{0}^{\infty}(\bar{\Omega})$ is dense in $\stackrel{\circ}{H}^{m}(\Omega)$. The rest of our task is to prove the completeness.

Let $\left\{u_{k}\right\}_{k=1,2, \ldots}$ be any Cauchy sequence in $\stackrel{\circ}{H}^{m}(\Omega)$. Since

$$
\left\|u_{k}-u_{l}\right\|_{L^{2}\left(\Omega_{R}\right)} \leqq C R\left\|\partial_{x}^{1}\left(u_{k}-u_{l}\right)\right\|_{L^{2}(\Omega)} \leqq C R\left|u_{k}-u_{l}\right|_{m, \Omega}
$$

as follows from Lemma 1.4, $\left\{u_{k}\right\}$ becomes a Cauchy sequence in $H^{m}\left(\Omega_{R}\right)$ for any $R \geqq r_{0}+1$. Let $u_{R}$ denote the limits of $\left\{u_{k}\right\}$ in $H^{m}\left(\Omega_{R}\right)$ and set $u(x)=u_{R}(x)$ for $x \in \Omega_{R}$. Then, $u(x)$ is well-defined as a function on $\Omega$ and $\in H_{\mathrm{loc}}^{m}(\Omega)$. It is easily seen that $\partial_{x}^{\alpha} u(x) \in L^{2}(\Omega)$ for $1 \leqq|\alpha| \leqq m$ and that

$$
\begin{aligned}
& \left|u_{k}-u\right|_{m, \Omega} \rightarrow 0, \\
& \left\|u_{k}-u\right\|_{L^{2}\left(\Omega_{R}\right.} \rightarrow 0 \quad \text { for any } \quad R \geqq r_{0}+1
\end{aligned}
$$

as $k \rightarrow \infty$. We must prove that $u(x)$ satisfies (1.1). Noting that $u_{k} \in \dot{H}^{1}(\Omega)$, by Lemma 1.3 we have

$$
\begin{aligned}
& \left\{R^{-2} \int_{R \leqq|x| \leqq 2 R}|u(x)|^{2} d x\right\}^{1 / 2} \\
& \leqq\left\{R^{-2} \int_{R \leqq|x| \leqq 2 R}\left|u(x)-u_{k}(x)\right|^{2} d x\right\}^{1 / 2}+4\left\{\int_{|x| \geqq R}\left|\partial_{x}^{1} u_{k}(x)\right|^{2} d x\right\}^{1 / 2} \\
& \leqq R^{-1}\left\|u-u_{k}\right\|_{L^{2}\left(\Omega_{2 R}\right)}+4\left|u_{k}-u\right|_{m, \Omega}+4\left\{\int_{|x| \geqq R}\left|\partial_{x}^{1} u(x)\right|^{2} d x\right\}^{1 / 2} .
\end{aligned}
$$

Letting $k \rightarrow \infty$ in (1.5) and using (1.4), we have

$$
R^{-2} \int_{R \leqq|x| \leqq 2 R}|u(x)|^{2} d x \leqq 16 \int_{|x| \geqq R}\left|\partial_{x}^{1} u(x)\right|^{2} d x .
$$

Since we already know that $\left|\partial_{x}^{1} u(x)\right| \in L^{2}(\Omega)$, it follows from (1.6) that $u$ satisfies (1.1), which completes the proof of the theorem.

Now, we shall prove Lemmas 1.2-1.4.

Proof of Lemma 1.2. Set $\chi_{r}(x)==\chi\left(r^{-1} x\right)$. Then, it follows that for some constant $C\left|\chi_{r} u--u\right|_{m, \Omega}^{2} \leqq C\left(I_{1} /(r)+I_{2}(r)\right)$ where

$$
\begin{aligned}
& I_{1}(r)=\sum_{\substack{1 \leqq|\alpha+\beta| \leqq m \\
|\beta| \geqq 1}} \int_{\Omega}\left|\partial_{x}^{\alpha}\left(1-\chi_{r}(x)\right) \partial_{x}^{\beta} u(x)\right|^{2} d x, \\
& I_{2}(r)=\sum_{1 \leqq|\alpha| \leqq m} \int_{\Omega}\left|\partial_{x}^{\alpha} \chi_{r}(x) u(x)\right|^{2} d x .
\end{aligned}
$$


Since $\partial_{x}^{\beta} u(x) \in L^{2}(\Omega)$ for $|\beta| \geqq 1$, it is seen that $I_{1}(r) \rightarrow 0$ as $r \rightarrow \infty$. Noting that

$$
I_{2}(r) \leqq \sum_{1 \leqq|\alpha| \leqq m} \sup \left|\partial_{x}^{\alpha} \chi\right|^{-2|\alpha|} \int_{r \leqq|x| \leqq 2 r}|u(x)|^{2} d x
$$

by (1.1) we have also that $l_{2}(r) \rightarrow 0$ as $r \rightarrow \infty$. These imply that for any integer $k \geqq 1$ there exists a $v_{k} \in H^{m}(\Omega)$ satisfying

$$
\left|u-v_{k}\right|_{m, \Omega} \leqq 1 / k \quad \text { and } \quad v_{k}(x)=u(x) \quad \text { for } \quad x \in \Omega_{R} .
$$

As is well-known, $C_{0}^{\infty}(\bar{\Omega})$ is dense in $H^{m}(\Omega)$. Thus, we can find $u_{k, R} \in C_{0}^{\infty}(\bar{\Omega})$ such that

$$
\sum_{|\alpha| \leqq m}\left\|\partial_{\alpha}^{x}\left(u_{k, R}-v_{k}\right)\right\|_{L^{2}(\Omega)} \leqq 1 / k
$$

Combining (1.7) and (1.8), we see easily that the sequence $\left\{u_{k, R}\right\}$ has the desired properties.

Proof of Lemma 1.3. First, we shall prove that

$$
\int_{|x| \geqq R}|\phi(x)|^{2}|x|^{-2 l} d x \leqq\left\{\frac{2}{n(1-(2 l / n))}\right\}_{|x| \geqq R}^{2}\left|\nabla_{x} \phi(x)\right|^{2}|x|^{-2(l-1)} d x
$$

for any $\phi \in C_{0}^{\infty}(\bar{\Omega}), R \geqq r_{0}$ and $l<n / 2$. We use the polar coordinates: $r=|x|$ and $\omega=x / r$. Since $|\phi(r \omega)|^{2} r^{-2 l}=-\int_{r}^{\infty} \frac{\partial}{\partial s}\left[|\phi(s \omega)|^{2} s^{-2 l}\right] d s$, we have

$$
\begin{aligned}
\int_{R}^{\infty}|\phi(r \omega)|^{2} r^{n-2 l-1} d r \leqq 2 \int_{R}^{\infty} r^{n-1} d r \int_{r}^{\infty}\left|\partial_{s} \phi(s \omega)\right||\phi(s \omega)| s^{-2 l} d s \\
+2 l \int_{R}^{\infty} r^{n-1} d r \int_{r}^{\infty}|\phi(s \omega)|^{2} s^{-2 l-1} d s .
\end{aligned}
$$

By integration by parts we have

$$
\begin{aligned}
& \text { (1.11) } 2 \int_{R}^{\infty} r^{n-1} d r \int_{r}^{\infty}\left|\partial_{s} \phi(s \omega)\right||\phi(s \omega)| s^{-2 l} d s \leqq \frac{2}{n} \int_{R}^{\infty}\left|\left(\nabla_{x} \phi\right)(r \omega)\right||\phi(r \omega)| r^{n-2 l} d r, \\
& \text { (1.12) } 2 l \int_{R}^{\infty} r^{n-1} d r \int_{r}^{\infty}|\phi(s \omega)|^{2} s^{-2 l-1} d s \leqq \frac{2 l}{n} \int_{R}^{\infty}|\phi(r \omega)|^{2} r^{n-2 l-1} d r
\end{aligned}
$$

By Schwarz's inequality we have

the right-hand side of (1.11)

$$
\leqq \frac{2}{n}\left\{\int_{R}^{\infty}\left|\left(\nabla_{x} \phi\right)(r \omega)\right|^{2} r^{n-2 l+1} d r\right\}^{1 / 2}\left\{\int_{R}^{\infty}|\phi(r \omega)|^{2} r^{n-2 l-1} d r\right\}^{1 / 2}
$$

Combining (1.10)-(1.13) implies that 


$$
(1-(2 l / n))\left\{\int_{R}^{\infty}|\phi(r \omega)|^{2} r^{2 n-2 l-1} d r\right\}^{1 / 2} \leqq \frac{2}{n}\left\{\int_{R}^{\infty}\left|\left(\nabla_{s} \phi\right)(r \omega)\right|^{2} r^{n-2 l+1} d r\right\}^{1 / 2}
$$

Since $1-(2 l / n)>0$, (1.9) follows immediately from (1.14). In particular, if we take $l=1$ (note that $n \geqq 3$ ), from (1.9) we have that (1.2) is valid for any $u \in C_{0}^{\infty}(\bar{\Omega})$.

Now, using Lemma 1.2, we shall prove that (1.2) is also valid for any $u \in \dot{H}^{1}(\Omega)$. Let $R^{\prime}$ be any number $>R$. By Lemma 1.2 we know that there exists a sequence $\left\{u_{k, R^{\prime}}\right\}_{k=1,2, \cdots} \subset C_{0}^{\infty}(\bar{\Omega})$ such that

$$
\left|u_{k, R^{\prime}}-u\right|_{1, \Omega} \rightarrow 0 \text { and }\left\|u_{k, R^{\prime}}-u\right\|_{L^{2}\left(\Omega_{R^{\prime}}\right)} \rightarrow 0 \quad \text { as } \quad k \rightarrow \infty .
$$

On the other hand, we have

$$
\begin{aligned}
& \left\{\int_{R \leqq|x| \leqq R^{\prime}}|u(x)|^{2}|x|^{-2} d x\right\}^{1 / 2} \\
& \leqq\left\{\int_{R \leqq|x| \leqq R^{\prime}}\left|u(x)--u_{k, R^{\prime}}(x)\right|^{2}|x|^{-2} d x\right\}^{1 / 2} \\
& +\left\{\int_{R \leqq|x| \leqq R^{\prime}}\left|u_{k, R^{\prime}}(x)\right|^{2}|x|^{-2} d x\right\}^{1 / 2} \\
& \leqq R^{-1}\left\|u_{k, R^{\prime}}-u\right\|_{L^{2}\left(\Omega_{R^{\prime}}\right)}+2\left|u_{k, R^{\prime}}-u\right|_{1, \Omega}+\left\{4 \int_{|x| \geqq R}\left|\partial_{x}^{1} u(x)\right|^{2} d x\right\}^{1 / 2} .
\end{aligned}
$$

Letting $k \rightarrow \infty$ in (1.16) and using (1.15), we have

$$
\int_{R \leqq 1 x \mid \leqq R^{\prime}}|u(x)|^{2}|x|^{-2} d x \leqq 4 \int_{|x| \geqq R}\left|\partial_{x}^{1} u(x)\right|^{2} d x .
$$

The arbitrariness of the choice of $R^{\prime}>R$ implies the lemma.

Proof of Lemma 1.4. In view of Lemma 1.3, it suffices to prove that there exists a constant $C>0$ such that

$$
\|u\|_{L^{2}\left(\Omega_{r_{0}+1}\right)} \leqq C\left\{\left\|\partial_{x}^{1} u\right\|_{L^{2}\left(\Omega_{r_{0}+1}\right)}+\|u\|_{L^{2}(G)}\right\}
$$

for any $u \in H^{1}\left(\Omega_{r_{0}+1}\right)$ where $G=\left\{x \in \mathbb{R}^{n}\left|r_{0} \leqq\right| x \mid \leqq r_{0}+1\right\}$. In fact, if (1.17) is valid, then we have

$$
\begin{aligned}
& \|u\|_{L^{2}\left(\Omega_{R}\right)}^{2}=\|u\|_{L^{2}\left(\Omega_{r_{0}+1}\right)}^{2}+\|u\|_{L^{2}\left(\left\{x \in \mathbb{R}^{n}\left|r_{0}+1 \leqq\right| x \mid \leqq R\right\}\right)}^{2} \\
& \quad \leqq 2 C^{2}\left\{\left\|\partial_{x}^{1} u\right\|_{L^{2}\left(\Omega_{r_{0}+1}\right)}^{2}+\left(r_{0}+1\right)^{2} \int_{G}|u(x)|^{2}|x|^{-2} d x\right\}+R^{2} \int_{r_{0}+1 \leqq|x| \leqq R}|u(x)|^{2}|x|^{-2} d x \\
& \quad \leqq\left(2 C^{2}+8 C^{2}\left(r_{0}+1\right)^{2}+4 R^{2}\right)\left\|\partial_{x}^{1} u\right\|_{L^{2}(\Omega)}^{2}
\end{aligned}
$$


for any $u \in \dot{H}^{1}(\Omega)$ and $R \geqq r_{0}+1$, from which the lemma follows.

Now, we prove (1.17). Suppose to the contrary that for any integer $k \geqq 1$ there exists a $u_{k} \in H^{1}\left(\Omega_{r_{0}+1}\right)$ such that

$$
\begin{aligned}
& \left\|u_{k}\right\|_{L^{2}\left(\Omega_{r_{0}+1}\right)}=1, \\
& \left\|\partial_{x}^{1} u_{k}\right\|_{L^{2}\left(\Omega_{r_{0}+1}\right)}+\left\|u_{k}\right\|_{L^{2}(G)}<1 / k .
\end{aligned}
$$

Since $\left\{u_{k}\right\}$ is a bounded sequence in $H^{1}\left(\Omega_{r_{0}+1}\right)$ as follows from (1.18) and (1.19), $\left\{u_{k}\right\}$ is weakly compact in $H^{1}\left(\Omega_{r_{0}+1}\right)$. Furthermore, since $\Omega_{r_{0}+1}$ is bounded, by Rellich's compactness theorem we see that $\left\{u_{k}\right\}$ is strongly compact in $L^{2}\left(\Omega_{r_{0}+1}\right)$. By passing to a subsequence if necessary, we can conclude that there exists a $v \in H^{1}\left(\Omega_{r_{0}+1}\right)$ such that

$$
\begin{aligned}
& u_{k} \rightarrow v \quad \text { weakly in } H^{1}\left(\Omega_{r_{0}+1}\right), \\
& u_{k} \rightarrow v \quad \text { strongly in } L^{2}\left(\Omega_{r_{0}+1}\right),
\end{aligned}
$$

as $k \rightarrow \infty$. It follows that from (1.19) and (1.20) that

$$
\left\|\partial_{x}^{1} v\right\|_{L^{2}\left(\Omega_{r_{0}+1}\right)}+\|v\|_{L^{2}(G)}=0
$$

which implies that $v=0$. In fact, the fact that $\partial_{x}^{1} v=0$ implies that $v$ is a constant vector. Thus, $|v|^{2} \times($ the Lebesgue measure of $G)=0$, from which we see that $v=0$.

On the other hand, (1.18) and (1.21) imply that the $L^{2}$ norm of $v$ on $\Omega_{r_{0}+1}$ is equal to 1 , which contradicts the fact that $v=0$. Thus, we have seen that (1.17) is valid, which completes the proof of the lemma.

Now, let us introduce the spaces and bilinear forms connected closely with the operator $L$ and the boundary condition. We define

$$
\begin{aligned}
& C_{B}^{\infty}(\bar{\Omega})=\left\{u \in C_{0}^{\infty}(\bar{\Omega}) \mid B L^{k} u=0 \text { on } \partial \Omega \text { fcr any } k \geqq 0\right\}, \\
& H_{B}^{\infty}(\Omega)=\left\{u \in \bigcap_{m=1}^{\infty} \dot{H}^{m}(\Omega) \mid B L^{k} u=0 \text { on } \partial \Omega \text { for any } k \geqq 0\right\}, \\
& H_{B}^{1}(\Omega)=\left\{\begin{array}{l}
\left\{u \in \dot{H}^{1}(\Omega) \mid u=0 \text { on } \partial \Omega\right\} \text { when } B u=\left.u\right|_{\partial \Omega}, \\
\dot{H}^{1}(\Omega) \text { when } B u=\left.\sum_{i, j=1}^{n} \nu_{i} a_{i j} \partial_{j} u\right|_{\partial \Omega},
\end{array}\right. \\
& H_{B}^{2}(\Omega)=\left\{u \in \dot{H}^{2}(\Omega) \mid B u=0 \text { on } \partial \Omega\right\}, \\
& \langle u, v\rangle_{1, \Omega}=\sum_{i, j, p, q=1}^{n} \int_{\Omega} a_{i p j q}(x) \partial_{j} u_{q}(x) \overline{\partial_{i} v_{p}(x)} d x, \\
& \langle u, v\rangle_{2, \Omega}=\langle u, v\rangle_{1, \Omega}+(L u, L v)_{L^{2}(\Omega)},
\end{aligned}
$$


where $u={ }^{t}\left(u_{1}, \cdots, u_{n}\right)$ and $v={ }^{t}\left(v_{1}, \cdots, v_{n}\right)$ and $(,)_{L^{2}(\Omega)}$ is the usual innerproduct of $L^{2}(\Omega)$.

First, we would like to prove that $\langle u, u\rangle_{1, \Omega}$ is the equivalent norm to $(u, u)_{1, \Omega}$. To do this, we need

Theorem 1.5 (Korn's first inequality). There exists a constant $\mathbb{C}>0$ such that

$$
C^{-1} \int_{\Omega}\left|\partial_{x}^{1} u\right|^{2} d x \leqq \sum_{i, j=1}^{n} \int_{\Omega}\left|\varepsilon_{i j}(u)\right|^{2} d x \leqq C \int_{\Omega}\left|\partial_{x}^{1} u\right|^{2} d x
$$

for any $u={ }^{t}\left(u_{1}, \cdots, u_{n}\right) \in \stackrel{\circ}{H}^{1}(\Omega)$, where $\varepsilon_{i j}(u)=\frac{1}{2}\left(\partial_{i} u_{j}+\partial_{j} u_{i}\right)$.

When $G$ is a bounded domain in $\mathbb{R}^{n}$, the following estimate is well-known as Korn's second inequality (cf. $\S 3$ of Duvaut and Lions [1] or Nitsche [11]):

$$
\int_{G}\left|\partial_{x}^{1} u(x)\right|^{2} d x \leqq C\left\{\sum_{i, j=1}^{n} \int_{G}\left|\varepsilon_{i j}(u)\right|^{2} d x+\int_{G}|u(x)|^{2} d x\right\}
$$

for any $u \in H^{1}(G)$. Friedrichs [2] also derived Korn's first inequality in the unbounded domain for the functions $u$ in the different classes. But, Theorem 1.5 does not follow directly from [2]. To prove Theorem 1.5 we need other ideas.

Proof of Theorem 1.5. It is trivial that the second part of the inequalities holds. Thus, we prove only the first part. Since $C_{0}^{\infty}(\bar{\Omega})$ is dense in $\dot{H}^{1}(\Omega)$ as follows from Theorem 1.1, we may assume that $u \in C_{0}^{\infty}(\bar{\Omega})$. Since by integration by parts we have for any $u \in \mathbb{C}_{0}^{\infty}\left(\mathbb{R}^{n}\right)$

$$
\sum_{i, j=1}^{n} \operatorname{Re} \int_{\mathbb{R}^{n}} \partial_{i} u_{j} \overline{\partial_{j} u_{i}} d x=\sum_{i, j=1}^{n} \int_{\mathbb{R}^{n}} \partial_{j} u_{j} \overline{\partial_{i} u_{i}} d x \geqq 0,
$$

it follows immediately that

$$
\sum_{i, j=1}^{n} \int_{\mathbb{R}^{n}}\left|\varepsilon_{i j}(u)\right|^{2} d x \geqq \frac{1}{2} \int_{\mathbb{R}^{n}}\left|\partial_{x}^{1} u\right|^{2} d x \quad \text { for any } u \in \mathbb{C}_{0}^{\infty}\left(\mathbb{R}^{n}\right)
$$

Using (1.22) and (1.23), we can prove that there exists a constant $C_{1}>0$ such that

$$
\left\|\partial_{x}^{1} u\right\|_{L^{2}(\Omega)}^{2} \leqq C_{1}\left\{\sum_{i, j=1}^{n} \int_{\Omega}\left|\varepsilon_{i j}(u)\right|^{2} d x+\|u\|_{L^{2}\left(\Omega_{r_{0}+1}\right)}^{2}\right\}
$$

for any $u \in C_{0}^{\infty}(\bar{\Omega})$. In fact, if we choose real-valued $C^{\infty}$ functions $\phi, \psi$ on $\mathbb{R}^{n}$ so that $\phi(x)=0$ for $|x| \geqq r_{0}+1, \psi(x)=0$ for $|x| \leqq r_{0}$ and $\phi^{2}(x)+\psi^{2}(x)=1$, then

$$
\left\|\partial_{x}^{1} u\right\|_{L^{2}(\Omega)}^{2} \leqq \int_{\Omega r_{0}+1}\left|\partial_{x}^{1}(\phi u)\right|^{2} d x+\int_{\mathbb{R}^{n}}\left|\partial_{x}^{1}(\psi u)\right|^{2} d x+\mathbb{C} \int_{\varrho r_{0}+1}|u|^{2} d x
$$


where $C=\sup \left|\nabla_{x} \phi\right|^{2}+\sup \left|\nabla_{x} \psi\right|^{2}$. Applying (1.22) and (1.23) and noting that $\left|\varepsilon_{i j}(\phi u)\right|^{2}+\left|\varepsilon_{i j}(\psi u)\right|^{2} \leqq 2\left(\phi^{2}+\psi^{2}\right)\left|\varepsilon_{i j}(u)\right|^{2}+4\left(\left|\nabla_{x} \phi\right|^{2}+\left|\nabla_{x} \psi\right|^{2}\right)|u|^{2}$ we have (1.24).

In view of (1.24), to complete the proof we have only to prove that there exists a constant $C_{2}>0$ such that

$$
\|u\|_{L^{2}\left(\Omega_{r_{0}+1}\right)} \leqq C_{2} \sum_{i, j=1}^{n} \int_{\Omega}\left|\varepsilon_{i j}(u)\right|^{2} d x \quad \text { for any } \quad u \in C_{0}^{\infty}(\bar{\Omega})
$$

Suppose to the contrary that for any integer $k \geqq 1$ there exists a $u_{k} \in C_{0}^{\infty}(\bar{\Omega})$ such that

$$
\begin{aligned}
& \left\|u_{k}\right\|_{L^{2}\left(\Omega_{r_{0}+1}\right)}=1 \\
& \sum_{i_{s} j=1}^{n} \int_{\Omega}\left|\varepsilon_{i j}\left(u_{k}\right)\right|^{2} d x<1 / k .
\end{aligned}
$$

Combining (1.24), (1.26) and (1.27), we see that $\left\{u_{k}\right\}_{k=1,2, \ldots}$ is a bounded set in $H^{1}\left(\Omega_{r_{0}+1}\right)$. Thus, by Rellich's compactness theorem we may assume that $\left\{u_{k}\right\}$ is a Cauchy sequence in $L^{2}\left(\Omega_{r_{0}+1}\right)$. Applying (1.24) to $u_{k}-u_{l}$ and using (1.27), we have

$$
\left|u_{k}-u_{l}\right|_{1, \Omega}^{2} \leqq C_{2}\left[(1 / k)+(1 / l)+\left\|u_{k}-u_{l}\right\|_{L^{2}\left(\Omega_{r_{0}+1}\right)}^{2}\right]
$$

This implies that $\left\{u_{k}\right\}$ is a Cauchy sequence in $\dot{H}^{1}(\Omega)$. By Theorem 1.1 we see that there exists a $w={ }^{t}\left(w_{1}, \cdots, w_{n}\right) \in \dot{H}^{1}(\Omega)$ such that $\left|u_{k}-w\right|_{1, \Omega} \rightarrow 0$ as $k \rightarrow \infty$. (1.27) implies that

$$
\partial_{i} w_{j}(x)+\partial_{j} w_{i}(x)=0 \quad \text { in } \Omega \text { for any } i, j=1, \cdots, n .
$$

Since $\partial_{i} \partial_{j} w_{k}=-\partial_{i} \partial_{k} w_{j}=\partial_{k} \partial_{j} w_{i}=-\partial_{i} \partial_{j} w_{k}$ as follows from (1.28), we see that $\partial_{i} \partial_{j} w_{k}=0$. These mean that $w_{k}$ are polynomials of order at most 1 . Noting that $\nabla_{x} w_{k} \in L^{2}(\Omega)$, we have that $w_{k}$ are constant. Then, we have

$$
R^{-2} \int_{R \leqq|x| \leqq 2 R}|w(x)|^{2} d x=|w|^{2} R^{n-2} c_{n}\left(2^{n}-1\right),
$$

where $c_{n}$ is the Lebesgue measure of the unit ball. Since $n \geqq 3$, by (1.1) we have that $w=0$. Namely, we can conclude that $\left|u_{k}\right|_{1, \Omega} \rightarrow 0$ as $k \rightarrow \infty$.

On the other hand, by (1.26) and Lemma 1.4 we have

$$
1=\left\|u_{k}\right\|_{L^{2}\left(\Omega_{r_{0}+1}\right)} \leqq C\left(r_{0}+1\right)\left|u_{k}\right|_{1, \Omega},
$$

which leads the contradiction. Thus, we have proved (1.25), which completes the proof. 
Corollary 1.6. Assume that (A.1) and (A.2) are valid. Then, there exists a constant $C>0$ such that

$$
C^{-1}|u|_{1, \Omega}^{2} \leqq\langle u, u\rangle_{1, \Omega} \leqq C|u|_{1, \Omega}^{2} \quad \text { for any } u \in \dot{H}^{1}(\Omega) .
$$

Proof. By (A.1) and (A.2) we have

$$
\langle u, u\rangle_{1, \Omega}^{2}=\sum_{i, j, p, q=1}^{n} \int_{\Omega} a_{i p j q} \varepsilon_{j q}(u) \overline{\varepsilon_{i p}(u)} d x \geqq \delta \sum_{i, j=1}^{n} \int_{\Omega}\left|\varepsilon_{i j}(u)\right|^{2} d x .
$$

Combining this and Theorem 1.5 implies that the first part of the inequalities is valid. The second part is trivial. The proof is complete.

When getting fundamental properties of the spaces $H_{B}^{1}(\Omega)$ and $H_{B}^{2}(\Omega)$ and proving the skew self-adjointness of $A$, the existence theorem of the solutions to the following problem plays an essential role:

$$
L u=v \text { in } \Omega, \quad B u=0 \text { on } \partial \Omega .
$$

To solve (1.29), first we consider the variational equation:

$$
\langle u, w\rangle_{1, \Omega}=-(v, w)_{L^{2}(\Omega)} \quad \text { for any } \quad w \in H_{B}^{1}(\Omega) .
$$

By Theorem 1.1 and Corollary 1.6 we see that $H_{B}^{1}(\Omega)$ is a Hilbert space equipped with the innerproduct $\langle,\rangle_{1, \Omega}$. It follows from Riesz's representation theorem that for any $v \in L_{R}^{2}(\Omega)=\left\{v \in L^{2}(\Omega) \mid v(x)=0\right.$ for $\left.|x|>R\right\}\left(R>r_{0}\right)$ there exists a unique $u \in H_{B}^{1}(\Omega)$ satisfying (1.30). Since

$$
\left|(v, w)_{L^{2}(\Omega)}\right| \leqq\|v\|_{L^{2}(\Omega)}\|w\|_{L^{2}\left(\Omega_{R}\right)} \leqq C R\|v\|_{L^{2}(\Omega)}|w|_{1, \Omega}
$$

as follows from Lemma 1.4, putting $u=w$ in (1.30) and using Corollary 1.6, we have

$$
|u|_{1, \Omega} \leqq C R\|v\|_{L^{2}(\Omega)} .
$$

Theorem 1.7. Assume that (A.1) and (A.2) are valid. Let $R$ be any number $>r_{0} . \quad$ If $v \in L_{R}^{2}(\Omega)$, then (1.29) admits a unique solution $u \in H_{B}^{2}(\Omega)$. In addition, we assume that $v \in H^{m}(\Omega)(m \geqq 1)$. Then, $u \in \stackrel{\circ}{H}^{m+2}(\Omega)$.

Furthermore, there exists a constant $C>0$ independent of $R, u$ and $v$ such that

$$
\sum_{|\alpha|=2}\left\|\partial_{x}^{\alpha} u\right\|_{L^{2}(\Omega)} \leqq C R\|v\|_{L^{2}(\Omega)} .
$$

Gilberg and Trudinger [3] obtain the same results as in this theorem in the case where $B u=\left.u\right|_{\partial \Omega}$ (cf. Theorems 8.8, 8.12 and 8.13 of [3]), and Shibata 
[12] in the case where $B u=\left.\sum \nu_{i} a_{i j} \partial_{j} u\right|_{\partial \Omega}$ (cf. Theorem 3.4 of [12]). Since, in the above discussion about the variational equation (1.30), we have got a unique $u$ in $H_{B}^{1}(\Omega)$ satisfying (1.30), the main part of the proof is to show that $\partial_{x}^{\alpha} u \in L^{2}(\Omega)$ for any $\alpha$ with $|\alpha|=2$. For detailed proof, see [3] and [12].

Using Corollary 1.6 and Theorem 1.7, we can prove

Lemma 1.8. Assume that (A.1) and (A.2) are valid. Then, there exists a constant $C>0$ such that

$$
C^{-1}|u|_{2, \Omega}^{2} \leqq\langle u, u\rangle_{2, \Omega} \leqq C|u|_{2, \Omega}^{2} \quad \text { for any } u \in H_{B}^{2}(\Omega) .
$$

Proof. The second part of the inequalities is trivial. Our task is to verify the first part. Choose $\phi \in C_{0}^{\infty}\left(\mathbb{R}^{n}\right)$ so that $\phi(x)=1$ for $|x| \leqq r_{0}+2$ and $=0$ for $|x| \geqq r_{0}+3$. For $u \in H_{B}^{2}(\Omega)$, put $v^{1}=-\phi L u, v^{2}=\phi L u-L(\phi u)$. Then, $v^{1}, v^{2} \in$ $L_{r_{0}+3}^{2}(\Omega)$, and by integration by parts we have

$$
\langle\phi u, w\rangle_{1, \Omega}=\left(v^{1}+v^{2}, w\right)_{L^{2}(\Omega)} \quad \text { for any } w \in H_{B}^{1}(\Omega) .
$$

The uniqueness of the solution of (1.30) and the estimates (1.31), (1.32) yield that

$$
\begin{aligned}
|\phi u|_{2, \Omega} & \leqq C_{1}\left(r_{0}+3\right)\left(\left\|v^{1}\right\|_{L^{2}(\Omega)}+\left\|v^{2}\right\|_{L^{2}(\Omega)}\right) \\
& \leqq C_{2}\left(\|L u\|_{L^{2}(\Omega)}+|u|_{1, \Omega}+\|u\|_{L^{2}\left(\Omega_{r_{0}+3}\right)}\right) .
\end{aligned}
$$

Next let us estimate $v=(1-\phi) u$. Noting that supp $[v] \subset\left\{x|| x \mid \geqq r_{0}+2\right\}$, by Corollary 1.6 and integration by parts we have for $i=1,2, \cdots, n$,

$$
\begin{aligned}
& \sum_{j=1}^{n}\left\|\partial_{j} \partial_{i} v\right\|_{L^{2}(\Omega)}^{2} \leqq C_{3}\left\langle\partial_{i} v, \partial_{i} v\right\rangle_{1, \Omega} \\
& =C_{3}\left\{\left(L v, \partial_{i}^{2} v\right)_{L^{2}(\Omega)}+\sum_{k, l, p_{q} q=1}^{n} \int_{\Omega}\left(\partial_{i} a_{k p l q}\right) \partial_{l} v_{q} \overline{\partial_{i} \partial_{k} v_{p}} d x\right\} \\
& \quad \leqq C_{4}\left\{\|L u\|_{L^{2}(\Omega)}+|u|_{1, \Omega}+\|u\|_{L^{2}\left(\Omega_{r_{0}+3}\right)}\right\} \sum_{|\alpha|=2}\left\|\partial_{x}^{\alpha} v\right\|_{L^{2}(\Omega)} .
\end{aligned}
$$

From this it follows that

$$
|(1-\phi) u|_{2, \Omega} \leqq C_{5}\left\{\|L u\|_{L^{2}(\Omega)}+|u|_{1, \Omega}+\|u\|_{L^{2}\left(\Omega_{r_{0}+3}\right)}\right\}
$$

Combining (1.33), (1.34) and Lemma 1.4, we obtain

$$
|u|_{2, \Omega} \leqq C_{6}\left\{\|L u\|_{L^{2}(\Omega)}+|u|_{1, \Omega}\right\} .
$$

From this and Corollary 1.6 the lemma follows.

Now, we summarize fundamental properties of the spaces $H_{B}^{1}(\Omega)$ and $H_{B}^{2}(\Omega)$. 
Theorem 1.9. Assume that (A.1) and (A.2) are valid. Then, $H_{B}^{1}(\Omega)$ and $H_{B}^{2}(\Omega)$ are Hilbert spaces equipped with the innerproducts $\langle,\rangle_{1, \Omega}$ and $\langle,\rangle_{2, \Omega}$, respectively.

Furthermore, $H_{B}^{\infty}(\Omega)$ and $C_{B}^{\infty}(\bar{\Omega})$ are dense in $H_{B}^{\infty}(\Omega)$ for $k=1,2$.

Proof. The first assertion immediately follows from Theorem 1.1, Corollary 1.6 and Lemma 1.8. Our task is to prove the seocnd assertion.

At first, we shall prove that $H_{B}^{\infty}(\Omega)$ is dense in $H_{B}^{1}(\Omega)$. Suppose to the contrary that there exists a non-trivial $u \in H_{B}^{1}(\Omega)$ such that

$$
\langle u, v\rangle_{1, \Omega}=0 \quad \text { for any } \quad v \in H_{B}^{\infty}(\Omega) .
$$

By Theorem 1.7, for any $w \in C_{0}^{\infty}(\Omega)$ we have a solution $v \in H_{B}^{\infty}(\Omega)$ of the equation: $L v=w$ in $\Omega$. By integration by parts we obtain

$$
\begin{aligned}
& \sum_{i, j, p, q=1}^{n} \int_{\Omega} \chi(x / R) a_{i p j q}(x) \partial_{j} u_{q}(x) \overline{\partial_{i} v_{p}(x)} d x \\
& =-(\chi(\cdot / R) u, L v)_{L^{2}(\Omega)}-\sum_{i, j, p, q=1}^{n} R^{-1} \int_{\Omega}\left(\partial_{j} \chi\right)(x / R) a_{i p j q}(x) u_{q}(x) \overline{\partial_{i} v_{p}(x)} d x
\end{aligned}
$$

for any $R>r_{0}$. Noting that $u$ satisfies (1.1) and letting $R \rightarrow \infty$ in (1.36), we get

$$
\langle u, v\rangle_{1, \Omega}=-(u, w)_{L^{2}(\Omega)} .
$$

Combining (1.35) and (1.37) yields that $(u, w)_{L^{2}(\Omega)}=0$ for any $w \in C_{0}^{\infty}(\Omega)$, which leads the contradiction. Thus, $H_{B}^{\infty}(\Omega)$ is dense in $H_{B}^{1}(\Omega)$.

As is easliy seen, proof of the fact that $C_{B}^{\circ}(\bar{\Omega})$ is dense in $H_{B}^{1}(\Omega)$ can be reduced to verifying that for any $v \in H_{B}^{\infty}(\Omega)|(1-\chi(\circ / R)) v|_{1, \Omega} \rightarrow 0$ as $R \rightarrow \infty$. This follows from the inequality:

$$
|(1-\chi(\cdot \mid R)) v|_{1, \Omega}^{2} \leqq \int_{R \leqq|x|}\left|\partial_{x}^{1} v\right|^{2} d x+\left(\sup \left|\nabla_{x} \chi\right|^{2}\right) R^{-2} \int_{R \leqq|x| \leqq 2 R}|v|^{2} d x,
$$

since $v$ satisfies the condition (1.1).

Next, let us prove that $H_{B}^{\infty}(\Omega)$ is dense in $H_{B}^{2}(\Omega)$. Suppose to the contrary that there exists a non-trivial $u \in H_{B}^{2}(\Omega)$ such that

$$
\langle u, v\rangle_{2, \Omega}=0 \quad \text { for any } v \in H_{B}^{\infty}(\Omega) \text {. }
$$

Introducing the innerproduct $\langle w, v\rangle_{1, \Omega}+(w, v)_{L^{2}(\Omega)}$ instead of $\left\langle u^{\prime}, v\right\rangle_{1, \Omega}$, in the similar manner to the proof of Theorem 1.7 we can prove that for any $w^{\prime} \in C_{0}^{\infty}(\Omega)$ there exists a $v \in H_{B}^{\infty}(\Omega)$ satisfying 


$$
-L v+v=w \quad \text { in } \Omega, \quad B v=0 \quad \text { on } \partial \Omega .
$$

In the same way as (1.37), (1.38) and (1.39) yield that $0=-\langle u, v\rangle_{2, \Omega}=$ $(L u, w)_{L^{2}(\Omega)}$ for any $w \in C_{0}^{\infty}(\Omega)$. This implies that $L u=0$ in $\Omega$. Hence, by the uniqueness of the solutions stated in Thoerem 1.7 we have $u(x)=0$ in $\Omega$, which leads the contradiction. Hence $H_{B}^{\infty}(\Omega)$ is dense in $H_{B}^{2}(\Omega)$.

The fact that $C_{B}^{\infty}(\bar{\Omega})$ is dense in $H_{B}^{2}(\Omega)$ can be proved in the same way as in $H_{B}^{1}(\Omega)$. Thus, we obtain the lemma.

When proving that $A$ is a skew self-adjoint operator, we need the following lemma which follows from Theorems 1.7 and 1.9.

Lemma 1.10. Assume that (A.1) and (A.2) are valid. Let $v \in L^{2}(\Omega)$. If $u \in H_{B}^{1}(\Omega)$ satisfies

$$
\langle u, w\rangle_{1, \Omega}=(v, w)_{L^{2}(\Omega)} \quad \text { for any } w \in C_{B}^{\infty}(\bar{\Omega}),
$$

then $u$ belongs to $H_{B}^{2}(\Omega)$.

Proof. Put $\chi_{R}(x)=\chi(x / R)$ for $R>r_{0}$. Then, it is obvious that $\chi_{R} u \rightarrow u$ in the disrtibution sense as $R \rightarrow \infty$. Therefore, if $\left\{\chi_{R} u\right\}_{R>r_{0}}$ is a bounded set in the Hilbert space $H_{B}^{2}(\Omega), u$ has to belong to $H_{B}^{2}(\Omega)$. For, there exists a subsequence of $\left\{\chi_{R} u\right\}$ converging weakly in $H_{B}^{2}(\Omega)$ and this limit coincides with the limit in the distribution sense.

Let us prove the boundedness of $\left\{\chi_{R} u\right\}$. By (1.40) and integration by parts we have

$$
\left\langle\chi_{R} u, w\right\rangle_{1, \Omega}=\left(\chi_{R} v+v_{R}^{\prime}, w^{\prime}\right)_{L^{2}(\Omega)} \quad \text { for any } w \in C_{B}^{\infty}(\bar{\Omega}),
$$

where $v_{R}^{\prime}=-\sum \partial_{i}\left(a_{i j}\left(\partial_{j} \chi_{R}\right) u\right)-\sum a_{i j}\left(\partial_{i} \chi_{R}\right) \partial_{j} u$. It is easy to see that $\chi_{R} u \in$ $H_{B}^{1}(\Omega)$ and $\chi_{R} v+\mathrm{v}_{R}^{\prime} \in L_{2 R}^{2}(\Omega)$ for any $R>r_{0}$. Since $C_{B}^{\infty}(\bar{\Omega})$ is dense in $H_{B}^{1}(\Omega)$ (cf. Theorem 1.9), (1.41) is valid for any $w \in H_{B}^{1}(\Omega)$. Therefore, from the uniqueness of the solution of (1.30) and Theorem 1.7 it follows that $\chi_{R} u \in H_{B}^{2}(\Omega)$ and $-L\left(\chi_{R} u\right)=\chi_{R} v+v_{R}^{\prime}$ for any $R>r_{0}$. By Lemma 1.8 we have

$$
\begin{aligned}
\left|\chi_{R} u\right|_{2, \Omega}^{2} & \leqq C_{1}\left(\left\|\chi_{R} v+v_{R}^{\prime}\right\|_{L^{2}(\Omega)}^{2}+\left|\chi_{R} u\right|_{1, \Omega}^{2}\right) \\
& \leqq C_{2}\left(\|v\|_{L^{2}(\Omega)}^{2}+|u|_{1, \Omega}^{2}+R^{-2} \int_{R \leqq|x| \leqq 2 R}|u(x)|^{2} d x\right),
\end{aligned}
$$

where the constants $C_{1}$ and $C_{2}$ are independent of $R>r_{0}$. Hence, by (1.1) we obtain the boundedness of $\left\{\chi_{R} u\right\}$ in $H_{B}^{2}(\Omega)$. The proof is complete.

From now on, we return to analysis of the operator $A$ (defined in (0.5)) and the equation (0.6). Put 


$$
H=H_{B}^{1}(\Omega) \times L^{2}(\Omega) \text { and }(f, g)_{E, \Omega}=\frac{1}{2}\left\{\left\langle f_{1}, g_{1}\right\rangle_{1, \Omega}+\left(f_{2}, g_{2}\right)_{L^{2}(\Omega)}\right\}
$$

for any $f=\left(f_{1}, f_{2}\right)$ and $g=\left(g_{1}, g_{2}\right) \in H$. As the innerproduct of $H$, we adopt $(,)_{E, \Omega^{*}}$ It is easily seen that $H$ is a Hilbert space, We introduce the following space as the domain of $A$ :

$$
D(A)=H_{B}^{2}(\Omega) \times\left\{H_{B}^{1}(\Omega) \cap L^{2}(\Omega)\right\},
$$

whose innerproduct is defined by

$$
(f, g)_{D(A), \Omega}=\frac{1}{2}\left\{\left\langle f_{1}, g_{1}\right\rangle_{2, \Omega}+\left\langle f_{2}, g_{2}\right\rangle_{1, \Omega}+\left(f_{2}, g_{2}\right)_{L^{2}(\Omega)}\right\}
$$

for $f=\left(f_{1}, f_{2}\right)$ and $g=\left(g_{1}, g_{2}\right) \in D(A)$. For the notational convenience, we define $A f$ by $A f=\left(f_{2}, L f_{1}\right)$ for $f=\left(f_{1}, f_{2}\right) \in D(A)$. It is obvious that $A f \in H$ if $f \in D(A) . \quad D(A)$ is a Hilbert space, and then, noting that $(f, g)_{D(A), \Omega}=(f, g)_{E, \Omega}$ $+(A f, A g)_{E, \Omega}$, we see that $A$ is a closed operator on $H$. Furthermore, it is seen from Theorem 1.9 that $A$ is densely defined in $H$.

Under these preparations, we shall prove the main result in this section.

Theorem 1.11. Assume that (A.1) and (A.2) are valid. Then, $A$ is a skew self-adjoint operator in $H$ with domain $D(A)$.

Proof. Let us recall the definition of the adjoint operator $A^{*}$ of $A$ and the domain $D\left(A^{*}\right)$ :

$$
\begin{aligned}
& D\left(A^{*}\right)=\{g \in H \mid \text { there exists an } h \in H \text { such that } \\
&\left.(g, A f)_{E, \Omega}=(h, f)_{E, \Omega} \text { for any } f \in D(A)\right\},
\end{aligned}
$$

and then for $g \in D\left(A^{*}\right) A^{*} g$ is defined by $A^{*} g=h$. Since integration by parts yields that $(g, A f)_{E, \Omega}=(-A g, f)_{E, \Omega}$ for any $f, g \in D(A)$, we know that $D(A) \subset$ $D\left(A^{*}\right)$. Our task is only to prove that $D\left(A^{*}\right) \subset D(A)$.

Let $g=\left(g_{1}, g_{2}\right)$ and $h=\left(h_{1}, h_{2}\right)$ be the elements in (1.42). Then, choosing $f=\left(f_{1}, f_{2}\right)$ so that $f_{1} \in H_{B}^{\infty}(\Omega)$ and $f_{2}=0$, by (1.42) we have

$$
\left\langle h_{1}, f_{1}\right\rangle_{1, \Omega}=\left(g_{2}, L f_{1}\right)_{L^{2}(\Omega)} \quad \text { for any } f_{1} \in H_{B}^{\infty}(\Omega) \text {. }
$$

From Theorem 1.7 it is seen that for any $v \in C_{0}^{\infty}(\Omega)$ there exists an $f_{1} \in H_{B}^{\infty}(\Omega)$ such that

$$
\mathbb{L} f_{1}=v \quad \text { in } \quad \& \text {. }
$$

Employing the same arguments as for (1.37), we have $\left\langle h_{1}, f_{1}\right\rangle_{1, \Omega}=-\left(h_{1}, L f_{1}\right)_{L}{ }^{2}(\Omega)$ 。 
Combining this, (1.43) and (1.44) implies that

$$
\left(g_{2}+h_{1}, v\right)_{L^{2}(\Omega)}=0 \quad \text { for any } v \in C_{0}^{\infty}(\Omega),
$$

which shows that $g_{2}=-h_{1} \in H_{B}^{1}(\Omega) \cap L^{2}(\Omega)$.

Next, choosing $f=\left(f_{1}, f_{2}\right)$ so that $f_{1}=0$ and $f_{2} \in C_{B}^{\infty}(\bar{\Omega})$, we have

$$
\left\langle g_{1}, f_{2}\right\rangle_{1, \Omega}=\left(h_{2}, f_{2}\right)_{L^{2}(\Omega)} \quad \text { for any } f_{2} \in C_{B}^{\infty}(\bar{\Omega}) .
$$

Since $g_{1} \in H_{B}^{1}(\Omega)$ and $h_{2} \in L^{2}(\Omega)$, by Lemma 1.10 we see that $g_{1} \in H_{B}^{2}(\Omega)$. Accordingly, we have proved that $g \in D(A)$, which completes the proof.

By Theorem 1.11 and Stone's theorem (cf. Appendix I in Lax and Phillips [6]), we have

Theorem 1.12. Assume that (A.1) and (A.2) are valid. Then, there exists a one parameter group $\{U(t)\}_{t \in \boldsymbol{R}}$ generated by $A$ and having the following properties:

(i) $U(t)$ is a unitary operator from $H$ to itself for any $t \in \boldsymbol{R}$.

(ii) $U(t) f$ is an $H$-valued continuous function in $t \in \boldsymbol{R}$ for any $f \in H$.

(iii) $U(t) f$ is an $H$-valued $C^{1}$ function in $t \in \boldsymbol{R}$ if and only if $f \in D(A)$.

(iv) $U(t)$ is a unitary operator also from $D(A)$ to itself for any $t \in \boldsymbol{R}$.

(v) When $f \in D(A), \frac{d}{d t} U(t) f=A U(t)=U(t)$ Af for any $t \in \boldsymbol{R}$.

From this theorem we see that for any $f=\left(f_{1}, f_{2}\right) \in D(A) u(t, x)=(U(t) f)_{1}(x)$ (= the first component of $U(t) f)$ belongs to $\bigcap_{j=0}^{2} C^{j}\left(\boldsymbol{R} ; H_{B}^{2-j}(\Omega)\right.$ ) $\left(H_{B}^{0}(\Omega)=L^{2}(\Omega)\right.$ ) and satisfies (0.1).

\section{§2. The Problem in the Free Space}

In this section we consider the unperturbed problem under the assumptions (A.1), (A.2) and (A.3) stated in $§ 0$ :

$$
\begin{cases}\left(\partial_{t}^{2}-L^{0}\right) u(t . x)=0 & \text { in } \boldsymbol{R} \times \boldsymbol{R}^{n}, \\ u(0, x)=f_{1}(x), \quad \partial_{t} u(0, x)=f_{2}(x) & \text { in } \boldsymbol{R}^{n}\end{cases}
$$

where $L^{0}=\sum_{i, j=1}^{n} a_{i j}^{0} \partial_{i} \partial_{j}$. Obviously, for this problem we can obtain the same result as in $\S 1$. We employ the same notations as in $\S 1$, and particularly we denote the space $H$ and the operators $U(t)$ for (2.1) by $H_{0}$ and $U_{0}(t)$, respectively. 
By (A.1) $L^{0}(\xi)$ is a symmetric matrix. So, all eigenvalues of $L^{0}(\xi)$ are real. Let $N$ denote the number of the distinct eigenvalues $\lambda_{j}(\xi)$ of $L^{0}(\xi)$. Then, by (A.3) $N$ is independent of $\xi \in \mathbb{R}^{n}-\{0\}$ and it can be shown that the $\lambda_{j}(\xi)$ can be enumerated so as to form $N$ distinct and analytic branches in the following way: $\lambda_{1}(\xi)<\lambda_{2}(\xi)<\cdots<\lambda_{N}(\xi)$. Since $L^{0}(t \xi)=t^{2} L^{0}(\xi), t \in \mathbb{R}$, with the enumeration used above the eigenvalues $\lambda_{j}(\xi)$ are $C^{\infty}$ functions homogeneous of order 2 in $\xi \in \mathbb{R}^{n}$. If we put $\varepsilon_{i p}=\frac{1}{2}\left(\xi_{i} \eta_{p}+\xi_{p} \eta_{i}\right)$ for $\xi=\left(\xi_{1}, \cdots, \xi_{n}\right)$ and $\eta=\left(\eta_{1}, \cdots, \eta_{n}\right)$ $\in \mathbb{R}^{n}$, by (A.1) and (A.2) we see that

$$
\eta L^{0}(\xi){ }^{t} \eta=\sum_{i, p, j, q=1}^{n} a_{i p j q}^{0} \varepsilon_{i q} \varepsilon_{j p} \geqq \delta_{1} \sum_{i, p=1}^{n}\left|\varepsilon_{i p}\right|^{2},
$$

where $a_{i p j q}^{0}$ are the $(p, q)$ elements of the matrices $a_{i j}^{0}$. Since $\sum_{i, p=1}^{n}\left|\varepsilon_{i p}\right|^{2} \geqq$ $\frac{1}{2}|\xi|^{2}|\eta|^{2}$, we see that there exists a $\delta>0$ such that

$$
\eta L^{0}(\xi){ }^{i} \eta \geqq \delta|\xi|^{2}|\eta|^{2} \quad \text { for any } \xi \text { and } \eta \in \mathbb{R}^{n} \text {. }
$$

From this we see that $\lambda_{j}(\xi) \geqq \delta|\xi|^{2}$ for any $\xi \in \mathbb{R}^{n}$. Let $\mathbb{P}_{j}(\xi)$ be the orthogonal projection into the eigenspace of each $\lambda_{j}(\xi)$, which an $n \times n$ matrix of $C^{\infty}$ functions on $\mathbb{R}^{n}-\{0\}$ homogeneous of order 0 .

The Radon transform $\tilde{g}(s, \omega)$ of $g(x) \in \mathcal{S}(\mathcal{S}$ is the Schwartz space of rapidly decreasing functions) is defined by

$$
\tilde{g}(s, \omega)=\int_{x \circ \omega=s} g(x) d S_{x}, \quad(s, \omega) \in \mathbb{R} \times \mathbb{S}^{n-1},
$$

where $S^{n-1}$ denotes the $n-1$ dimensional unit sphere. We denote the Fourier transform of $g(x)\left(x \in \mathbb{R}^{n}\right)$ by $\mathscr{F}[g](\xi)=\hat{g}(\xi)\left(=\int e^{-i x \cdot \xi} g(x) d x, i=\sqrt{-1}\right)$, and the one of $k(s)(s \in \mathbb{R})$ by $F[k](\sigma)\left(=\int e^{-i s \sigma} k(s) d s\right)$. Let their inverse transformations be denoted by $\mathscr{F}^{-1}$ and $F^{-1}$, respectively. Then, it follows that

$$
\begin{aligned}
& g(x)=2^{-1}(2 \pi)^{1-n} \int_{S^{n-1}} F^{-1}\left(|\sigma|^{n-1} F \tilde{g}\right)(x \circ \omega, \omega) d \omega, \\
& \hat{g}(\sigma \omega)=F \tilde{g}(\sigma, \omega) .
\end{aligned}
$$

Set

$$
\lambda_{ \pm}(\sigma)=\left\{\begin{array}{cl}
\frac{1-i}{\sqrt{2}} \sigma^{1 / 2} & \text { for } \sigma \geqq 0 \\
\pm \frac{1+i}{\sqrt{2}}|\sigma|^{1 / 2} & \text { for } \sigma<0
\end{array}\right.
$$


Then, we see that $\operatorname{supp}\left[\lambda_{+}\left(D_{s}\right) k\right] \subset\left(-\infty, s_{0}\right]$ (resp. supp $\left.\left[\lambda_{-}\left(D_{s}\right) k\right] \subset\left[s_{0}, \infty\right)\right)$ provided that supp $[k] \subset\left(-\infty, s_{0}\right]\left(\right.$ resp. $\left.\subset\left[s_{0}, \infty\right)\right)$ and that $\left(\lambda_{ \pm}\left(D_{s}\right)\right)^{2}=-\partial_{s}$, where $\lambda_{ \pm}\left(D_{s}\right) k=F^{-1}\left[\lambda_{ \pm}(\sigma) F k(\sigma)\right]$ (cf. Lemma 1.1 of Soga [13]). Setting

$$
J_{ \pm}= \begin{cases}\left(-\partial_{s}\right)^{(n-1) / 2} & \text { for odd } n, \\ \left(-\partial_{s}\right)^{(n / 2)-1} \lambda_{ \pm}\left(D_{s}\right) & \text { for even } n\end{cases}
$$

for the data $f=\left(f_{1}, f_{2}\right) \in \mathcal{S}$ in (2.1) we define $T_{0}^{ \pm} f$ by

$$
T_{0}^{ \pm} f(s, \omega)=\sum_{j=1}^{N} \lambda_{j}(\omega)^{1 / 4} P_{j}(\omega)\left(-\lambda_{j}(\omega)^{1 / 2} \partial_{s} J_{ \pm} \tilde{f}_{1}+J_{ \pm} \tilde{f}_{2}\right)\left(\lambda_{j}(\omega)^{1 / 2} s, \omega\right)
$$

Then, $f(x) \in \mathcal{S}$ is reconstructed with $T_{0}^{ \pm} f(s, \omega)$ as follows:

Theorem 2.1. For any $f=\left(f_{1}, f_{2}\right) \in \mathcal{S}$ we have

$$
\begin{aligned}
& f_{1}(x)=-2^{-1}(2 \pi)^{1-n} \int_{S^{n-1}} \sum_{j=1}^{N} \lambda_{j}(\omega)^{-n / 4} P_{j}(\omega)\left(\partial_{s}^{-1} J_{ \pm}^{*} T_{\overline{0}}^{-} f\right)\left(\lambda_{j}(\omega)^{-1 / 2} x \cdot \omega, \omega\right) d \omega \\
& f_{2}(x)=2^{-1}(2 \pi)^{1-n} \int_{S^{n-1}} \sum_{j=1}^{N} \lambda_{j}(\omega)^{-n / 4} P_{j}(\omega)\left(J_{ \pm}^{*} T_{0}^{ \pm} f\right)\left(\lambda_{j}(\omega)^{-1 / 2} x \cdot \omega, \omega\right) d \omega
\end{aligned}
$$

The above formulas are corresponding to Theorem 2.2 in Chapter IV of Lax and Phillips [6] (cf. Proposition 1.1 of Soga [13] also). Noting that $P_{j}(\omega)$ are the orthogonal projections, we can derive the above theorem from (2.2) in the same way as in [6] and [13].

For any $k(s, \omega) \in \mathcal{S}\left(\mathbb{R} \times S^{n-1}\right)$ we define

$$
\left(Q_{0}^{ \pm} k\right)(x)=2^{-1}(2 \pi)^{1-n} \int_{S^{n-1}} \sum_{j=1}^{N} \lambda_{j}(\omega)^{-n / 4} P_{j}(\omega)\left(\partial_{s}^{-1} J_{ \pm}^{*} k\right)\left(\lambda_{j}(\omega)^{-1 / 2} x \cdot \omega, \omega\right) d \omega,
$$

(2.8) $Q^{ \pm} k=\left(-Q_{0}^{ \pm} k, Q_{0}^{ \pm}\left(\partial_{s} k\right)\right)$.

Obviously $\left(Q^{ \pm} k\right)(x)$ belong to $C^{\infty}\left(\mathbb{R}^{n}\right)$; Theorem 2.1 means that $Q^{ \pm} T_{0}^{ \pm} f=f$ for any $f \in \mathcal{S}$. Furthermore, we obtain

Lemma 2.2. Set $\mathcal{S}^{\circ}=\left\{k(s, \omega) \in \mathcal{S}\left(\mathbb{R} \times S^{n-1}\right) \mid F k(\sigma, \omega)=0\right.$ in a neighborhood of $\sigma=0\}$. Then, for any $k \in \mathcal{S}$ we have $\left(Q^{ \pm} k\right)(x) \in \mathcal{S}$ and $T_{0}^{ \pm} Q^{ \pm} k=k$.

Proof. For $k(s, \omega) \in \stackrel{\mathcal{S}}{\text { set }}$

$$
\stackrel{\circ}{k}(\sigma, \omega)=\sum_{j=1}^{N} \lambda_{j}(\omega)^{(2-n) / 4} P_{j}(\omega) F\left[\partial_{s}^{-1} J_{ \pm}^{*} k\right]\left(\lambda_{j}(\omega)^{1 / 2} \sigma, \omega\right) .
$$

Then $\stackrel{\circ}{k}(\sigma, \omega)$ belongs to $\mathcal{S}\left(\mathbb{R} \times S^{n-1}\right)$ and is equal to 0 in a neighborhood of $\sigma=0$. Furthermore, it follows that

$$
\left(Q_{0}^{\mp} k\right)(x)=2^{-1} \mathscr{F}^{-1}\left[\{\hat{k}(|\xi|, \xi /|\xi|)+\hat{k}(-|\xi|,-\xi|| \xi \mid)\}|\xi|^{1-n}\right](x),
$$


which yields that $\left(Q^{ \pm} k\right)(x) \in \mathcal{S}$.

Combining (2.3), (2.9) and the definitions (2.7), (2.8) and noting that $P_{j}(\omega)$ are the orthogonal projections, we have $T_{0}^{ \pm} Q^{ \pm} k(s, \omega)=\sum_{j=1}^{4} \Phi_{j}(s, \omega)$ where

$$
\begin{gathered}
\Phi_{1}(s, \omega)=\frac{1}{2} \sum_{j=1}^{N} \lambda_{j}(\omega)^{3 / 4} P_{j}(\omega) F^{-1}\left[K_{1}(\cdot, \omega)\right]\left(\lambda_{j}(\omega)^{1 / 2} s\right), \\
K_{1}(\sigma, \omega)=\grave{k}(\sigma, \omega)|\sigma|^{1-n} i \sigma J_{ \pm}(\sigma), \\
\Phi_{2}(s, \omega)=\frac{1}{2} \sum_{j=1}^{N} \lambda_{j}(\omega)^{1 / 4} P_{j}(\omega) F^{-1}\left[K_{2}(\cdot, \omega)\right]\left(\lambda_{j}(\omega)^{1 / 2} s\right), \\
K_{2}(\sigma, \omega)=\left(\partial_{s} k^{0}\right)(\sigma, \omega)|\sigma|^{1-n} J_{ \pm}(\sigma), \\
\Phi_{3}(s, \omega)=\frac{1}{2} \sum_{j=1}^{N} \lambda_{j}(\omega)^{3 / 4} P_{j}(\omega) F^{-1}\left[K_{3}(\cdot, \omega)\right]\left(\lambda_{j}(\omega)^{1 / 2} s\right), \\
K_{3}(\sigma, \omega)=k_{4}(-\sigma,-\omega)|\sigma|^{1-n} i \sigma J_{ \pm}(\sigma), \\
K_{4}(\sigma, \omega)=\partial_{s} k^{0}(-\sigma,-\omega)|\sigma|^{1-n} i \sigma J_{ \pm}(\sigma) .
\end{gathered}
$$

Noting that $F\left[\partial_{s}^{-1} J_{ \pm}^{*} k\right](\sigma, \omega)=(i \sigma)^{-1} \overline{J_{ \pm}(\sigma)}[F k](\sigma, \omega)$ and that $J_{ \pm}(\sigma) \overline{J_{ \pm}(\sigma)}=|\sigma|^{n-1}$, by the change of the variable: $\lambda_{l}(\omega)^{1 / 2} \sigma \rightarrow \sigma$, we have

$$
F^{-1}\left[K_{1}(\cdot, \omega)\right]\left(\lambda_{j}(\omega)^{1 / 2} s\right)=\sum_{l=1}^{N} \lambda_{l}(\omega)^{-3 / 4} P_{l}(\omega) k\left(\lambda_{l}(\omega)^{-1 / 2} \lambda_{j}(\omega)^{1 / 2} s, \omega\right) .
$$

Using the fact that the $P_{j}(\omega)$ are the orthogonal projections again, it follows that $\Phi_{1}(s, \omega)=2^{-1} k(s, \omega)$. In the same way, we also obtain $\Phi_{2}=\Phi_{1}$ and $\Phi_{3}=-\Phi_{4}$. These prove that $\left(T_{0}^{ \pm} Q^{ \pm} k\right)(s, \omega)=k(s, \omega)$, which completes the proof of the lemma.

Lax and Phillips [6] say that a unitary operator $T_{0}^{+}$(resp. $T_{0}^{-}$) from $H_{0}$ to $L^{2}\left(\boldsymbol{R} \times S^{n-1}\right)$ is the outgoing (resp. incoming) translation representation if there exists a closed subspace $D_{0}^{+}$(resp. $D_{0}^{-}$) in $H_{0}$ such that

$$
\begin{aligned}
& \text { (2.10) } T_{0}^{ \pm} \text {map } D_{0}^{ \pm} \text {onto } L_{ \pm}^{2}\left(\boldsymbol{R} \times S^{n-1}\right)=\left\{k(s, \omega) \in L^{2}\left(\boldsymbol{R} \times S^{n-1}\right) \mid k(s, \omega)=0\right. \\
& \text { for } \pm s<0\}, \\
& \text { (2.11) } U_{0}^{ \pm}(t) D_{0}^{ \pm} \subset D_{0}^{ \pm} \quad \text { for } \pm t>0, \\
& \text { (2.12) } \bigcap_{t \in \mathbb{R}} U_{0}(t) D_{0}^{ \pm}=\{0\},
\end{aligned}
$$$$
\text { (2.13) } \overline{\bigcup_{t \in \mathbb{R}} U_{0}(t) D_{0}^{ \pm}}=H_{0} \text {, }
$$

and $T_{0}^{ \pm}$satisfy 


$$
T_{0}^{ \pm} U_{0}(t)=\mathscr{I}_{t} T_{0}^{ \pm},
$$

where $\left(\mathscr{I}_{t} k\right)(s)=k(s-t)$. And, $D_{0}^{+}$(resp. $D_{0}^{-}$) is called the outgoing (resp. incoming) subspace. The operator $T_{0}^{+}$(resp. $T_{0}^{-}$) defined by (2.4) becomes the outgoing (resp. incoming) translation representation:

Theorem 2.3. (i) The operators $T_{0}^{ \pm}$(in (2.4)) can be extended as unitary operators from $H_{0}$ to $L^{2}\left(\boldsymbol{R} \times S^{n-1}\right)$.

(ii) Let us denote these extentions of $T_{0}^{ \pm}$also by $T_{0}^{ \pm}$. Set $D_{0}^{ \pm}=\left\{f \in H_{0} \mid\right.$ $T_{0}^{ \pm} f(s, \omega)=0$ when $\left.\pm_{-}<0\right\}$. Then, $T_{0}^{+}$(resp. $T_{0}^{-}$) is the outgoing (resp. incoming) translation representation, and $D_{0}^{+}$(resp. $\left.D_{0}^{-}\right)$is the outgoing (resp. incoming) subspace (i.e., all the conditions (2.10)-(2.14) are satisfied).

Proof. (i) Noting that $P_{j}(\omega)$ are the orthogonal projections, for any $f=\left(f_{1}, f_{2}\right) \in \mathcal{S}$ we have

$$
\begin{aligned}
& \left\|T_{0}^{ \pm} f\right\|_{L^{2}\left(\boldsymbol{R} \times S^{n-1}\right)}^{2}=\iint_{\boldsymbol{R} \times S^{n-1}} \sum_{j=1}^{N}\left|\lambda_{j}^{1 / 2} P_{j} \partial_{s} J_{ \pm} \tilde{f}_{1}\right|^{2} d s d \omega \\
& \quad+\iint_{\boldsymbol{R} \times S^{n-1}} \sum_{j=1}^{N}\left|P_{j} J_{ \pm} \tilde{f}_{2}\right|^{2} d s d \omega-2 \operatorname{Re} \iint_{\boldsymbol{R} \times S^{n-1}} \sum_{j=1}^{N} \lambda_{j}^{1 / 2} P_{j} \partial_{s} J_{ \pm} \tilde{f}_{1} \cdot \overline{P_{j} J_{ \pm}} \tilde{f}_{2} d s d \omega .
\end{aligned}
$$

Therefore, using the facts that $P_{j}(\omega)=P_{j}(-\omega), F \tilde{g}(\sigma, \omega)=F \tilde{g}(-\sigma,-\omega)$ and that $\sum_{j=1}^{N} \sigma^{2} \lambda_{j}(\omega) P_{j}(\omega)=L^{0}(\sigma \omega)$, we have

$$
\left\|T_{0}^{ \pm} f\right\|_{L^{2}\left(\boldsymbol{R} \times S^{n-1}\right)}^{2}=4(2 \pi)^{n-1}\|f\|_{E, \boldsymbol{R}^{n}}^{2} \quad \text { for any } f \in \mathcal{S} .
$$

Let us take $2^{-1}(2 \pi)^{(1-n) / 2}\|k\|_{L^{2}\left(\boldsymbol{R} \times S^{n-1}\right)}$ as the norm of $k \in L^{2}\left(\boldsymbol{R} \times S^{n-1}\right)$. Then, $T_{0}^{ \pm}$can be regarded as isometric operators from $H_{0}$ to $L^{2}\left(\boldsymbol{R} \times S^{n-1}\right)$, because $\mathcal{S}\left(\supset C_{0}^{\infty}\left(\boldsymbol{R}^{n}\right)\right.$ ) is dense in $H_{0}$ (cf. Theorem 1.9). Lemma 2.2 yields that $Q^{ \pm} \dot{\mathcal{S}} \subset \mathcal{S}$ and $\mathcal{S} \subset T_{0}^{ \pm} \mathcal{S}$. Hence, the (extended) operators $T_{0}^{ \pm}$map $H_{0}$ onto $L^{2}\left(\boldsymbol{R} \times S^{n-1}\right)$ since $\mathcal{S}^{\circ}$ is dense in $L^{2}\left(\boldsymbol{R} \times S^{n-1}\right)$. Thus, (i) of the theorem is proved.

(ii) From (i) of the theorem we see easily that the spaces $D_{0}^{ \pm}$are closed subspaces in $H_{0}$ and mapped onto $L_{ \pm}^{2}\left(\boldsymbol{R} \times S^{n-1}\right)$ by $T_{0}^{ \pm}$(i.e., (2.10) is satisfied).

Let us check (2.14). Assume that the data $f=\left(f_{1}, f_{2}\right)$ in (2.1) belong to $C_{0}^{\infty}\left(\boldsymbol{R}^{n}\right)$. Then, from finiteness of the propagation speed (cf. Theorem 3.1 in $\S 3$ below), it is seen that the solution $u(t, x)$ is a $C_{0}^{\infty}\left(\boldsymbol{R}_{x}^{n}\right)$-valued $C^{\infty}$ function on $\boldsymbol{R}_{t}$. Therefore, for any fixed $t$ the concrete definition form of the Radon transform $\tilde{u}(t, s, \omega)$ is valid. Noting that $\tilde{\partial_{i}} u(t, s, \omega)=\omega_{i} \partial_{s} \tilde{u}(t, s, \omega)$ and $L^{0}(\omega)=\sum_{j=1}^{N} \lambda_{j}(\omega) P_{j}(\omega)\left(\omega \in S^{n-1}\right)$, we have $\left(\partial_{t}^{2} u-L^{0} u\right)^{\sim}(s, \omega)=\sum_{j=1}^{N}\left(\partial_{t}^{2}-\lambda_{j}(\omega) \partial_{s}^{2}\right)$ 
$P_{j}(\omega) \tilde{u}(t, s, \omega)$. If we put $v_{j}(t, s, \omega)=\left(\partial_{t}-\lambda_{j}(\omega)^{1 / 2} \partial_{s}\right) P_{j}(\omega) \tilde{u}(t, s, \omega)$, then $\left(\partial_{t}+\lambda_{j}(\omega)^{1 / 2} \partial_{s}\right) v_{j}(t, s, \omega)=0$ for any $(t, s, \omega) \in \mathbb{R} \times \mathbb{R} \times S^{n-1}$ and $j=1, \cdots, N$, which yields that $v_{j}(t, s, \omega)=v_{j}\left(0, s-\lambda_{j}(\omega)^{1 / 2} t, \omega\right)$. Combining this and the fact that $P_{j}(\omega)\left(T_{0}^{ \pm} U_{0}(t) f\right)(s, \omega)=\lambda_{j}(\omega)^{1 / 4}\left(J_{ \pm} v_{j}\right)\left(t, \lambda_{j}(\omega)^{1 / 2} s, \omega\right)$, we obtain $P_{j}(\omega)$ - $\left(T_{0}^{ \pm} U_{0}(t) f\right)(s, \omega)=P_{j}(\omega)\left(T_{0}^{ \pm} f\right)(s-t, \omega)$ for any $(s, t, \omega) \in \mathbb{R} \times \mathbb{R} \times S^{n-1}$ and $j=1, \cdots, N$, which proves $(2.14)$ (note that $C_{0}^{\infty}\left(\mathbb{R}^{n}\right)$ is dense in $\left.H_{0}\right)$.

Finally we have only to verify (2.11)-(2.13). From what we have proved, we see that (2.11)-(2.13) mean that $\mathscr{I}_{t} L_{ \pm}^{2}\left(\mathbb{R} \times S^{n-1}\right) \subset L_{ \pm}^{2}\left(\mathbb{R} \times S^{n-1}\right)( \pm t>0)$, $\cap \mathscr{I}_{t} L_{ \pm}^{2}\left(\mathbb{R} \times S^{n-1}\right)=0$ and $\bar{\cup} \mathscr{I}_{t} L_{ \pm}^{2}\left(\mathbb{R} \times S^{n-1}\right)=L^{2}\left(\mathbb{R} \times S^{n-1}\right)$, respectively. These are obvious. The proof is complete.

$D_{0}^{ \pm}$are characterized in the following theorem. This characterization plays a fundamental role when defining the translation representations for the mixed problem (0.1) in $\S 3$ below.

Theorem 2.4. f belongs to $D_{0}^{ \pm}$if and only if

$$
\operatorname{supp}\left[U_{0}(t) f\right] \subset\{(t, x)| \pm \eta t \leqq| x \mid\},
$$

where $\eta=\min \left\{\lambda_{j}(\omega)^{1 / 2} \mid \omega \in S^{n-1}, j=1, \cdots, N\right\}$.

Proof. The idea of the proof is due to Lax and Phillips [6, 7] (cf. Theorem 1.2 in Chapter VI of [6] and Corollary 4.2 of [7]). We shall give only an outline of the proof.

Let $f \in D_{0}^{ \pm}$. Then there exists a sequence $\left\{k_{ \pm}^{i}\right\}_{i=1,2, \ldots}$ in $C_{0}^{\infty}\left(\mathbb{R} \times S^{n-1}\right)$ such that $\operatorname{supp}\left[k_{ \pm}^{i}\right] \subset \mathbb{R}_{ \pm} \times S^{n-1}\left(\mathbb{R}_{ \pm}=\{s \in \mathbb{R} \mid \pm s>0\}\right)$ and $\left\|k_{ \pm}^{i}-T_{\overline{0}}^{ \pm} f\right\|_{L^{2}\left(\mathbb{R} \times S^{n-1}\right)} \rightarrow 0$ as $i \rightarrow \infty$. In the same way as in Soga [13] (see Corollary 1.1 of [13]), we see that the formulas (2.5) and (2.6) are valid for $f=f_{ \pm}^{i}=\left(T_{0}^{ \pm}\right)^{-1} k_{ \pm}^{i}$. Therefore, combining (2.5), (2.6) and (2.14), we have

$$
\left(U_{0}(t) f_{ \pm}^{i}\right)_{l}(x)=\frac{1}{2}(2 \pi)^{1-n} \sum_{j=1}^{N} \int \lambda_{j}(\omega)^{-n / 4} P_{j}(\omega)\left(\partial_{s}^{l-2} J_{ \pm}^{*} k_{ \pm}^{i}\right)\left(\lambda_{j}(\omega)^{-1 / 2} x \cdot \omega-t, \omega\right) d \omega
$$

for $l=1$ and 2 . From these formulas and the fact that supp $\left[\partial_{s}^{l-2} J_{ \pm}^{*} k_{ \pm}^{i}\right] \subset$ $\overline{\mathbb{R}}_{ \pm} \times S^{n-1}$, it follows that $\operatorname{supp}\left[U_{0}(t) f_{ \pm}^{i}\right] \subset\left\{(t, x) \in \mathbb{R}^{n+1}| \pm \eta t \leqq| x \mid\right\}$ for $\pm t>0$. On the other hand, for any fixed $t U_{0}(t) f_{ \pm}^{i}$ converge to $U_{0}(t) f$ in $L_{\text {loc }}^{2}\left(\mathbb{R}^{n}\right)$ as $i \rightarrow \infty$. Hence, we obtain (2.15).

Conversely, let $(2.15)$ be satisfied. We can assume without loss of generality that $f \in D\left(A_{0}^{\infty}\right) \underset{m=1}{=} \cap D_{0}^{\infty}\left(A_{0}^{m}\right)$ (the operator $A$ is denoted by $A_{0}$ here), which means that $\partial_{s}^{m} T_{0}^{ \pm} f(s, \omega) \in L^{2}\left(\right.$ 职 $\left.\times S^{n-1}\right)$ for any $m \geqq 1$. Therefore, $J_{ \pm}^{*} T_{0}^{ \pm} f(s, \omega)$ is $L^{2}\left(S_{\omega}^{n-1}\right)$-valued (consequently $L^{1}\left(S_{\omega}^{n-1}\right)$-valued) $C^{\infty}$ functions in 
$s \in \boldsymbol{R}$. The proof can be reduced to verifying that

$$
\begin{array}{r}
\left(\partial_{x}^{\alpha} U_{0}(t) f\right)_{2}(0)=\frac{1}{2}(2 \pi)^{1-n}\left(-\partial_{t}\right)^{|\alpha|} \int_{S^{n-1}} \sum_{j=1}^{N}\left(\lambda_{\jmath}(\omega)^{-1 / 2} \omega\right)^{\alpha} \\
\cdot \lambda_{j}(\omega)^{-n / 4} P_{j}(\omega)\left(J_{ \pm}^{*} T_{0}^{ \pm} f\right)(-t, \omega) d \omega
\end{array}
$$

for any multi-index $\alpha$ (note that $U_{0}(t) f(x) \in C^{\infty}$, which follows from $f \in$ $D\left(A_{0}^{\infty}\right)$ ). In fact, since $\partial_{x}^{\alpha} U_{0}(t) f(0)=0$ for any $\pm t>0$, by (2.16) we have

$$
\int_{S^{n-1}} \sum_{j=1}^{N}\left(\lambda_{j}(\omega)^{-1 / 2} \omega\right)^{\alpha} \lambda_{j}(\omega)^{-n / 4} P_{j}(\omega)\left(J_{ \pm}^{*} T_{\overline{0}}^{-\perp} f\right)(-t, \omega) d \omega=0
$$

for any $\alpha$ and $\pm t>0$. Combining this fact and Lemma 1.1 in Chapter VI of Lax and Phillips [6] yields that $P_{j}(\omega)\left(J_{ \pm}^{*} T_{0}^{ \pm} f\right)(s, \omega)=0$ for any $\pm s<0, \omega \in S^{n-1}$ and $j=1, \cdots, N$, which proves that $f \in D_{0}^{ \pm}$.

(2.16) can be verified as follows. Combining (2.6) and (2.14), we can easily obtain (2.16) if $f \in \mathcal{S}$. For $f \in D\left(A_{0}^{\circ}\right)$, take a sequence $\left\{f^{i}\right\}_{i=1,2, \ldots} \subset \mathcal{S}$ such that $\sum_{k=0}^{m}\left\|A_{0}^{k}\left(f^{i}-f\right)\right\|_{E, \boldsymbol{R}^{n} \rightarrow 0}$ as $i \rightarrow \infty(m>|\alpha|+n / 2)$. Then, it is seen that for any fixed $t U_{0}(t) f^{i} \rightarrow U_{0}(t) f$ in $H_{\mathrm{loc}}^{m}\left(\mathbb{R}^{n}\right)$ as $i \rightarrow \infty$, and that $T_{0}^{ \pm} f^{i}(s, \omega) \rightarrow T_{0}^{ \pm} f(s, \omega)$ in $H^{m}\left(\mathbb{R}_{s} ; L^{2}\left(S_{\omega}^{n-1}\right)\right)$ as $i \rightarrow \infty$. These facts imply that $(2.16)$ is valid for any $f \in D\left(A_{0}^{\infty}\right)$.

\section{§. The Problem in the Exterior Domain}

Throughout this section we use the notations in $\S \S 1$ and 2 . It is expected that the problem $(0.1)$ has finite propagation speed: There exists a constant $\mu(>0)$ such that $u\left(t_{0}, x_{0}\right)=0$ when $u(T, x)=0$ for any $x \in\left\{y \in \Omega|| y-x_{0} \mid<\right.$ $\left.\mu\left(t_{0}-T\right)\right\}\left(T<t_{0}\right)$. This is derived from the following local energy estimate.

Theorem 3.1. Let the assumptions (A.1) and (A.2) be satisfied. Then, there exists a constant $\mu(>0)$ depending only on $n, \sup _{x \in \Omega}\left|a_{i j}(x)\right|$ and the constant $\delta$ in (A.2) such that if $u(t, x) \in C^{2}(\mathbb{R} \times \bar{\Omega})$ is a solution of $(0.1)$, for any $T>0$, $R>0$ and $y^{\prime} \in \bar{\Omega} U(t) f\left(=\left(u\left(t, x,\left(\partial_{t} u(t, x)\right)\right)\right.\right.$ satisfies

$$
\|U(T) f\|_{E, \Omega_{R}(y)} \leqq\|f\|_{E, \Omega_{R+\mu_{T}(y)}},
$$

where $\Omega_{R}(y)=\{x \in \Omega|| x-y \mid<R\}$ and

$$
\|f\|_{E, G}^{2}=\frac{1}{2} \int_{G}\left\{\sum_{i, j, p, q=1}^{n} a_{i p j q}(x) \partial_{j} f_{1 q}(x) \overline{\partial_{i} f_{1 p}(x)}+\left|f_{2}(x)\right|^{2}\right\} d x
$$

$\left(f=\left(f_{1}, f_{2}\right)\right.$ and $\left.f_{l}={ }^{t}\left(f_{l 1}, \cdots, f_{l n}\right), l=1,2\right)$. 
Proof. The idea of the proof is the same as in the proof of Theorem 1.1 in Chapter V of Lax and Phillips [6]. Multiply the equation $\left(\partial_{i}^{2}-L\right) u=0$ by $\overline{\partial_{t} u}$ and integrate over $D=\{(t, x)|0<t<T| x-y \mid,<R+(T-t) \mu, x \in \Omega\}$. Then, by integration by parts we have

$$
\begin{gathered}
\|U(T) f\|_{E, \Omega_{R}(y)}^{2}-\|U(0) f\|_{E, \Omega_{R+} \mu_{T}(y)}^{2}+\int_{((0, T) \times \partial \Omega) \cap D}\left(\partial_{t} u \cdot \overline{N u}+N u \cdot \overline{\partial_{t} u}\right) d t d(\partial \Omega) \\
+\left(1+\mu^{2}\right)^{-1 / 2} \int_{\Gamma}\left\{\mu\left(\left|\partial_{t} u\right|^{2}+\sum_{i, j, p, q=1}^{n} a_{i p j q} \partial_{j} u_{q} \cdot \overline{\partial_{i} u_{p}}\right)\right. \\
\left.-2 \operatorname{Re} \sum_{i, p, j, q=1}^{n} a_{i p j q} r_{i} \partial_{j} u_{q} \cdot \overline{\partial_{t} u_{p}}\right\} d \Gamma=0,
\end{gathered}
$$

where $\Gamma=\{(t, x)|0<t<T| x-y \mid,=R+(T-t) \mu, x \in \Omega\}, r_{i}=\left(x_{i}-y_{i}\right) /|x-y|$ and $N u=\sum_{i, j=1}^{n} \nu_{i} a_{i j} \partial_{j} u$. Therefore, if $u$ satisfies (0.1), it follows from (A.1) and (A.2) that

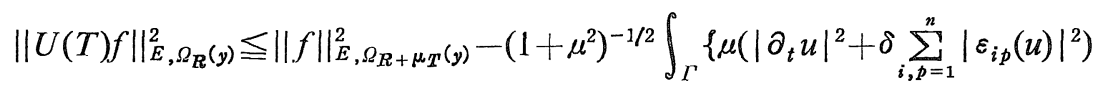

$$
\begin{aligned}
& \left.-n^{2}\left(\sup _{i, j=1, \cdots, n}\left|a_{i j}\right|\right)\left(n\left|\partial_{t} u\right|^{2}+\sum_{i, p=1}^{n}\left|\varepsilon_{i p}(u)\right|^{2}\right)\right\} d \Gamma \text {. }
\end{aligned}
$$

Take the $\mu$ so that $\mu \geqq\left(\max \left\{n^{3}, n^{2} / \delta\right\}\right)$ sup $\left|a_{i j}\right|$. Then, the integral over $\Gamma$ in (3.2) is non-negative, and consequently (3.1) is obtained. The proof is complete.

Hereafter, we assume that the coefficients of $L$ in (0.1) are constant (i.e., $L=L^{0}$ ), and that (A.1), (A.2) and (A.3) in $\S 0$ are satisfied. From now on, using the translation representations $T_{0}^{ \pm}$in the free space, we make the translation representations for the perturbed equation (0.1) in the same way as in $\S 2$ of Chapter V of Lax and Phillips [6]. Set

$$
D_{ \pm}=U_{0}\left( \pm r_{0} \eta^{-1}\right) D_{0}^{ \pm}
$$

Then, it follows from Theorem 2.4 that if $f \in D_{ \pm}, U_{0}(t) f(x)=0$ in a neighborhood of $\partial \Omega$ for any $\pm t>0$. Therefore, $D_{ \pm}$become closed subspaces in $H$, and we have

$$
U_{0}(t) f=U(t) f \quad \text { for any } \quad \pm t>0 \quad \text { if } f \in D_{ \pm} .
$$

Combining this and Theorem 2.3, we obtain easily (i) and (ii) in the following theorem.

Theorem 3.2. $D_{+}$(resp. D_) is an outgoing (resp. incoming) subspace for $U(t)$ : 
(i) $U(t) D_{ \pm} \subset D_{ \pm}( \pm t>0)$,

(ii) $\bigcap_{t \in \mathbb{R}} U(t) D_{ \pm}=\{0\}$,

(iii) $\overline{\bigcup_{t \in R} U(t) D_{ \pm}}=H$.

The (iii) of Theorem 3.2 is a key point, and is closely related to the local energy decay as was stated in $\S 2$ of Chapter $V$ of Lax and Phillips [6]. We shall prove (iii) later, and we use this theorem without the proof for a while. For any $f \in \bigcup_{t \in R} U(t) D_{ \pm}$we set

$$
T_{ \pm} f=\lim _{\tau \rightarrow \infty} \mathcal{I}_{\mp \tau \mp r_{0} \eta^{-1}} T_{0}^{ \pm} U(\tau) f .
$$

Then, $T_{ \pm}$are densely defined in $H$ (from (iii) of Theorem 3.2 and (3.3)), and can be regarded as isometric operators from $H$ to $L^{2}\left(\boldsymbol{R} \times S^{n-1}\right)$ (by Theorem 2.3). Furthermore, we obtain

Corollary 3.3. $T_{+}$(resp. $T_{-}$) is the outgoing (resp. incoming) translation representation for $U(t)$ with the outgoing (resp. incoming) subspace $D_{+}$(resp. $D^{-}$).

In the same way as in Remark 2.2 in Chapter V of Lax and Phillips [6], we see from (iii) of Theorem 3.2 also that the wave operators:

$$
W_{ \pm}=\lim _{t \rightarrow \pm \infty} U(-t) U_{0}(t)
$$

are well-defined and complete. Namely, we have

Corollary 3.4. $W_{ \pm}$are unitary operators from $H_{0}$ to $H$.

Note that the scattering operator $S=\left(T_{+}\right)^{-1} T_{-}$is well-defined and unitary from $L^{2}\left(\boldsymbol{R} \times S^{n-1}\right)$ to itself.

Now, we shall give a proof of Theorem 3.2. As we mentioned earlier, it suffices to prove only (iii) of Theorem 3.2. Lax and Phillips in $\S 2$ of Chapter $\mathrm{V}$ of [6] showed that (iii) could be derived from the non-existence of the point spectrum of $A$ and the local energy estimate stated in Theorem 3.1. Thus, the proof is complete if the following theorem is verified.

Theorem 3.5. A has no point spectrum. Namely, if $f \in D(A)$ and $A f=\tau f$ for $a \tau \in \boldsymbol{C}$ (= the field of complex numbers), then $f=0$.

To prove this theorem, we need the following lemma.

Lemma 3.6. Let $\sigma \in \boldsymbol{R}-\{0\}$. If $u \in L^{2}\left(\boldsymbol{R}^{n}\right)$ satisfies: $\left(L^{0}+\sigma^{2} I\right) u(x)=0$ for $|x|>R$ where $I$ is the $n \times n$ identity matrix, then $u(x)=0$ for $|x|>R$. 
The proof of this lemma will be reduced to the following theorem due to Hörmander [4], Littman [8] and Murata [10].

Theorem 3.7. Let the polynomial $P(\xi)$ in $\xi \in \mathbb{R}^{n}$ be decomposed into the form: $c \prod_{j=1}^{l} P_{j}(\xi)^{\beta_{j}}\left(c \in \mathbb{C}\right.$ and $\beta_{j}$ are positive integers), where (i) $P_{j}(\xi)(j=1, \cdots, l)$ are irreducible and real polynomials,

(ii) there exists a $\xi^{j} \in \mathbb{R}^{n}$ for each $P_{j}$ such that $P_{j}\left(\xi^{j}\right)=0$ and $\operatorname{grad} P_{j}\left(\xi^{j}\right) \neq 0$. If $\phi \in L_{\mathrm{loc}}^{2}\left(\mathbb{R}^{n}\right) \cap \mathcal{S}^{\prime}\left(\mathcal{S}^{\prime}\right.$ is the dual space of $\left.\mathcal{S}\right)$ satisfies

$$
\lim _{R \rightarrow \infty} R^{-1} \int_{R \leqq|x| \leqq 2 R}|\phi(x)|^{2} d x=0
$$

and supp $\left[P\left(D_{x}\right) \phi\right]$ is compact $\left(D_{x}=\frac{1}{i}\left(\partial_{1}, \cdots, \partial_{n}\right)\right)$, then supp $[\phi]$ is compact; more precisely, supp $[\phi] \subset$ the convex hull of supp $\left[P\left(D_{x}\right) \phi\right]$.

Proof of Lemma 3.6. Let $u={ }^{t}\left(u_{1}, \cdots, u_{n}\right)$ and set $P(\xi)=$ the determinat of the matrix $\sigma^{2} I-L^{0}(\xi)$. Miultiplying $L^{0}+\sigma^{2} I$ by its cofactor, we see that $P\left(D_{x}\right) u_{j}(x)=0$ for $|x|>R$. Therefore, if we check that $P(\xi)$ has all the properties stated in Theorem 3.7, the lemma follows from this theorem, since $\phi=$ $u_{j} \in L^{2}\left(\mathbb{R}^{n}\right)$ satisfies (3.4).

Let us check that $P(\xi)$ satisfies (i) and (ii) of Theorem 3.7. Put $\xi=\left(\xi^{\prime}, \xi_{n}\right)$ $\in \mathbb{R}^{n-1} \times \mathbb{R}$. For $j=1, \cdots, N$ we have $\sigma^{2}-\lambda_{j}\left(0, \xi_{n}^{j}\right)=0$ and $\left(\partial \lambda_{j} / \partial \xi_{n}\right)\left(0, \xi_{n}^{j}\right) \neq 0$ where $\xi_{n}^{j}=|\sigma| \lambda_{j}(0,1)^{-1 / 2}$ by the Euler identity. Hence, there exist positivevalued $C^{\infty}$ functions $\eta^{j}\left(\xi^{\prime}\right)$ defined in a neighborhood $\omega$ of $\xi^{\prime}=0$ such that $\sigma^{2}-\lambda_{j}\left(\xi^{\prime}, \eta^{j}\left(\xi^{\prime}\right)\right)=0$ for any $\xi^{\prime} \in \omega$ and $\eta^{j}(0)=\xi_{n}^{j}$. It is seen that $P(\xi)$ is of the form:

$$
P\left(\xi^{\prime}, \xi_{n}\right)=\left(\operatorname{det} a_{n n}^{0}\right) \prod_{j=1}^{N}\left(\xi_{n}-\eta^{j}\left(\xi^{\prime}\right)\right)^{\alpha}{ }_{j}\left(\xi_{n}+\eta^{j}\left(\xi^{\prime}\right)\right)^{\alpha},
$$

where $\alpha_{j}$ are the multiplicities of $\lambda_{j}(\xi)$ and det $a_{n n}^{0}$ denotes the determinant of the matrix $a_{n n}^{0}$. Note that det $a_{n n}^{0}>0$, which follows from (A.1) and (A.2). Decompose $P(\xi)$ into the product of the irreducible polynomials $P_{j}(\xi), j=1, \cdots, l$, i.e., $P(\xi)=\prod_{j=1}^{l} P_{j}(\xi)^{\beta_{j}}$. Then, the set $\left\{\mu_{k}^{j}\left(\xi^{\prime}\right) \mid k=1, \cdots, m_{j}, j=1, \cdots, l\right\}$ of the roots of the equations: $P_{j}\left(\xi^{\prime}, \xi_{n}\right)=0$ in $\xi_{n}(j=1, \cdots, l)$ coincides with the set $\left\{- \pm \eta^{j}\left(\xi^{\prime}\right) \mid j=1, \cdots, N\right\}$ for any $\xi^{\prime}$ in an open set $\subset \omega$. From this fact we can see that every $P_{j}(\xi)$ satisfies (i) and (ii) in Theorem 3.7, which completes the proof of the lemma.

Proof of Theorem 3.5. Since $0=(A f, f)_{E, \Omega}+(f, A f)_{E, \Omega}=2 \operatorname{Re} \tau\|f\|_{E, \Omega}^{2}$ for 
any $f \in D(A)$ satisfying: $A f=\tau f$, the theorem is trivial when $\operatorname{Re} \tau \neq 0$. Let $\tau=i \sigma(\sigma \in \mathbb{R})$. Then, from the equation: $A f=i \sigma f\left(f=\left(f_{1}, f_{2}\right) \in D(A)\right)$ we have

$$
\begin{aligned}
& \left(L^{0}+\sigma^{2} I\right) f_{1}(x)=0 \quad \text { in } \Omega, \\
& f_{2}=i \sigma f_{1} .
\end{aligned}
$$

If $\sigma=0$, then $f_{2}=0$ and $L^{0} f_{1}=0$ in $\Omega$. The uniqueness of the solutions (cf. Theorem 1.7) implies that $f_{1}=0$, and then $f=0$. If $\sigma \neq 0$, it follows from (3.5) that $f_{1}=-\sigma^{-2} L^{0} f_{1} \in L^{2}(\Omega)$. Thus, if we put $u(x)=\chi\left(x / r_{0}\right) f_{1}(x)$ for $x \in \Omega$ and $=0$ for $x \notin \Omega$, then $u \in L^{2}\left(\mathbb{R}^{n}\right)$ and $\left(L^{0}+\sigma^{2} I\right) u(x)=0$ for $|x|>2 r_{0}$. Hence, by Lemma 3.6 we have $u(x)=0$ for $|x|>2 r_{0}$, which means that

$$
f_{1}(x)=0 \text { for }|x|>2 r_{0} .
$$

Note that $L^{0}+\sigma^{2} I$ is strongly elliptic. Thus, the analytic-hypoellipticity implies that $f_{1}(x)$ is analytic in $\Omega$. It follows from (3.7) that $f_{1}(x)=0$ for all $x \in \Omega$. Consequently, we have $f=0$, which completes the proof.

\section{References}

[1] Duvaut, G. and Lions, J.L., Les inéquations en mécanique et en physique, Dunot, 1972.

[2] Friedrichs, K.O., On the boundary value problems of the theory of elasticity and Korn's inequality, Ann. of Math., 48 (1947), 441-471.

[3] Gilberg, D. and Trudinger, N.S., Elliptic partial differential equations of second order, Grund. math. Wiss., 224, Springer, 1977.

[4] Hörmander, L., Lower bounds at infinity for solutions of differeníal equations with constant coefficients, Israel J. Math., 16 (1973), 103-116.

[5] Iwashita, H. and Shibata, Y., On the analyticity of spectral functions for some exterior boundary value problem, to appear in Glasnik Mat.

[6] Lax, P.D. and Phillips, R.S., Scattering theory, Academic Press, New York, 1967.

[7] - Scattering theory for the acoustic equation in an even number of space dimensions, Indiana Univ. Math. J., 22 (1972), 101-134.

[8] Littman, W., Decay at infinity of solutions of partial differential equations; removal of the curvature assumptions, Israel J. Math., 8 (1970), 403-407.

[9] Marsden, J.E. and Hughes, T.J.R., Mathematical foundations of elasticity, PrenticeHall, Englewood Cliffs, 1983.

[10] Murata, M., Asymptotic behaviors at infinity of solutions of certain linear partial differential equations, J. Fac. Sci. Univ. Tokyo, Sec. IA, 23 (1976), 107-148.

[11] Nitsche J.A., On Korn's second inequality, RAIRO, Analyse Numérique, 15(3) (1983), 237-248.

[12] Shibata, Y., On the Neumann problem for some linear hyperbolic systems of second order, preprint in 1987.

[13] Soga, H., Singularities of the scartering kernel for convex obstacles, J. Math. Kyoto Univ., 22 (1983), 729-765.

[14] Yamamoto, $\mathbb{K}$., Exponential energy decays of solutions of elastic wave equations with Dirichlet condition, to appear in Math. Scand. 
